\title{
Transcription factor RUNX1 promotes survival of acute myeloid leukemia cells
}

\author{
Susumu Goyama, ${ }^{1}$ Janet Schibler, ${ }^{1}$ Lea Cunningham, ${ }^{2}$ Yue Zhang, ${ }^{1}$ Yalan Rao, ${ }^{1}$ Nahoko Nishimoto, ${ }^{3}$ \\ Masahiro Nakagawa, ${ }^{3}$ Andre Olsson, ${ }^{4}$ Mark Wunderlich, ${ }^{1}$ Kevin A. Link, ${ }^{1}$ Benjamin Mizukawa, ${ }^{1}$ \\ H. Leighton Grimes, ${ }^{1,4}$ Mineo Kurokawa, ${ }^{3}$ P. Paul Liu, ${ }^{2}$ Gang Huang, ${ }^{1}$ and James C. Mulloy ${ }^{1}$
}

\begin{abstract}
1Division of Experimental Hematology and Cancer Biology, Cincinnati Children's Hospital Medical Center, University of Cincinnati College of Medicine, Cincinnati, Ohio, USA. ${ }^{2}$ Oncogenesis and Development Section, Center for Cancer Research, National Cancer Institute, NIH, Bethesda, Maryland, USA. ${ }^{3}$ Department of Hematology and Oncology, Graduate School of Medicine, University of Tokyo, Tokyo, Japan. ${ }^{4}$ Division of Immunobiology, Cincinnati Children's Hospital Medical Center, Cincinnati, Ohio, USA.
\end{abstract}

\begin{abstract}
RUNX1 is generally considered a tumor suppressor in myeloid neoplasms. Inactivating RUNX1 mutations have frequently been found in patients with myelodysplastic syndrome (MDS) and cytogenetically normal acute myeloid leukemia (AML). However, no somatic RUNX1 alteration was found in AMLs with leukemogenic fusion proteins, such as core-binding factor (CBF) leukemia and MLL fusion leukemia, raising the possibility that RUNX1 could actually promote the growth of these leukemia cells. Using normal human cord blood cells and those expressing leukemogenic fusion proteins, we discovered a dual role of RUNX1 in myeloid leukemogenesis. RUNX1 overexpression inhibited the growth of normal cord blood cells by inducing myeloid differentiation, whereas a certain level of RUNX1 activity was required for the growth of AML1-ETO and MLL-AF9 cells. Using a mouse genetic model, we also showed that the combined loss of Runx1/Cbfb inhibited leukemia development induced by MLL-AF9. RUNX2 could compensate for the loss of RUNX1. The survival effect of RUNX1 was mediated by BCL2 in MLL fusion leukemia. Our study unveiled an unexpected prosurvival role for RUNX1 in myeloid leukemogenesis. Inhibiting RUNX1 activity rather than enhancing it could be a promising therapeutic strategy for AMLs with leukemogenic fusion proteins.
\end{abstract}

\section{Introduction}

RUNX1 (also called AML1) is a member of the RUNX transcription factor family and plays an essential role in the development of normal hematopoiesis $(1,2)$. RUNX1 forms a core-binding factor (CBF) complex with its cofactor, CBFB. RUNX1 and CBFB are the most frequent targets of chromosomal translocations in acute myeloid leukemia (AML), generating the leukemogenic fusion proteins AML1-ETO and CBFB-MYH11. In these CBF leukemias, the dominant inhibition of RUNX1 function by these fusion proteins has been considered a critical pathway for leukemia development (3). In MLL fusion leukemia, RUNX1 expression is downregulated through degradation by MLL fusion proteins (ref. 4 and G. Huang, unpublished observations). Furthermore, inactivating RUNX1 mutations have been frequently found in a variety of myeloid neoplasms, including myelodysplastic syndrome (MDS) and cytogenetically normal AML (5-8). Therefore, RUNX1 has been regarded as a beneficial tumor suppressor for myeloid leukemogenesis. The tumor suppressor activity of RUNX1 has also been demonstrated in several mouse AML models. Runx1-deficient cells showed increased susceptibility to AML development in collaboration with MLL-ENL, NRASG12S, and EVI5 (9-11).

However, human genetic evidence also raises the possibility that RUNX1 activity is required for optimal growth of AML cells. RUNX1 mutation is usually heterozygous, and complete loss of RUNX1 is rarely seen in human AMLs. In particular, no RUNX1 mutation was found in AMLs with leukemogenic fusion proteins, such as CBF and MLL fusion leukemias $(7,8,12)$. Experimentally, it was shown that $C b f b-M y b 11$ knockin mice developed leukemia partly through RUNX1 repression-independent activities, and

Conflict of interest: The authors have declared that no conflict of interest exists. Citation for this article: J Clin Invest. 2013;123(9):3876-3888. doi:10.1172/JCI68557. a mutant CBFB-MYH11 lacking the RUNX1 binding domain induced leukemia quickly despite its inefficient suppression of RUNX1 function $(13,14)$.

We have developed an experimental system to model myeloid leukemogenesis using primary human cord blood (CB) cells $(15,16)$. The CBF fusion proteins AML1-ETO and CBFB-MYH11 promote self-renewal and long-term proliferation of human $\mathrm{CB} \mathrm{CD} 34^{+}$cells in vitro (17-19). The MLL fusion protein MLL-AF9 immortalizes $\mathrm{CB}$ cells in vitro and produces human leukemia in immunodeficient mice (20). These engineered pre-leukemic and leukemic cells recapitulate many features of the clinical diseases and have been useful in testing different therapeutic strategies (21-25).

Using these human cell-based models, we identified a contextspecific, dual role for RUNX1 in human myeloid neoplasms. Consistent with the general assumption, RUNX1 induces myeloid differentiation in normal CB cells, thereby working as a tumor suppressor. Unexpectedly, we also found that RUNX1 plays a critical role in the growth and survival of human AML cells. We used a mouse model of AML, which demonstrated that the combined deletion of Runx1/Cbfb diminishes the leukemogenic activity of murine MLL-AF9 cells. Our study uncovers a prosurvival role for RUNX1 in myeloid leukemia and identifies RUNX1 as a potential therapeutic target beyond CBF leukemia.

\section{Results}

RUNX1 inbibits the growth of buman CB cells. We first examined the effect of forced expression of RUNX1 and its mutants in human $\mathrm{CB} \mathrm{CD} 34^{+}$cells. R139G and D171N have a point mutation in the RUNT domain. S291fsX9 (S291fs) is a C-terminal truncation mutant lacking an activation domain. L378fsX196 $\mathrm{Ex} 7$ (L378fs) is a C-terminal elongation mutant and lacks sequences of exon 7. These mutations were identified exclusively in MDS/AML patients 
A

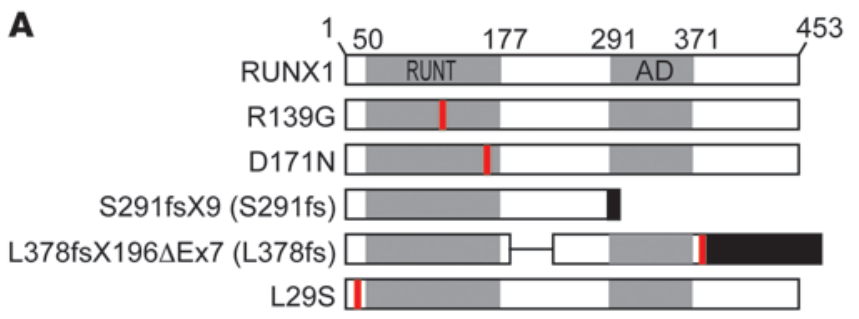

C

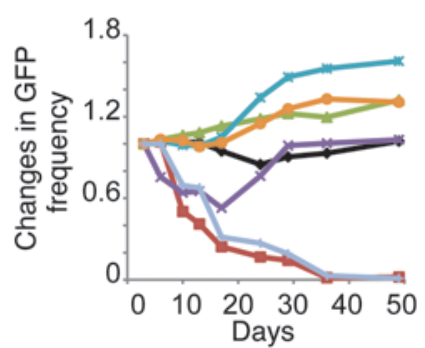

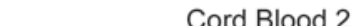

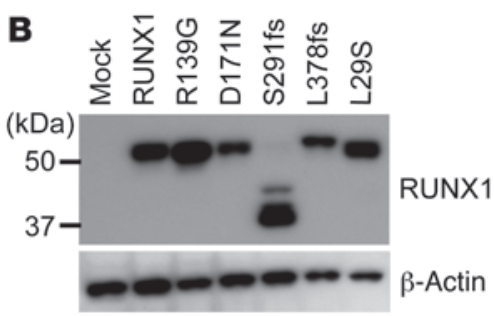

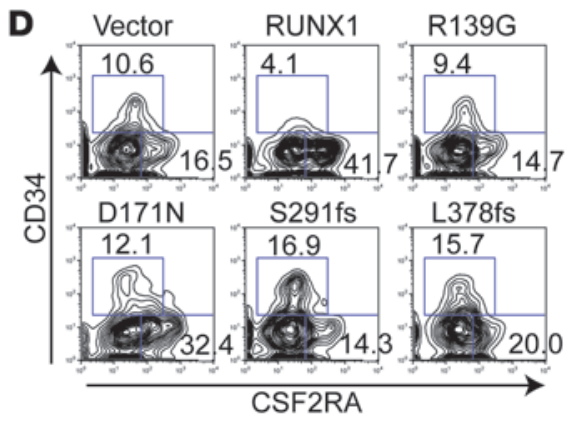

$\mathbf{E}$
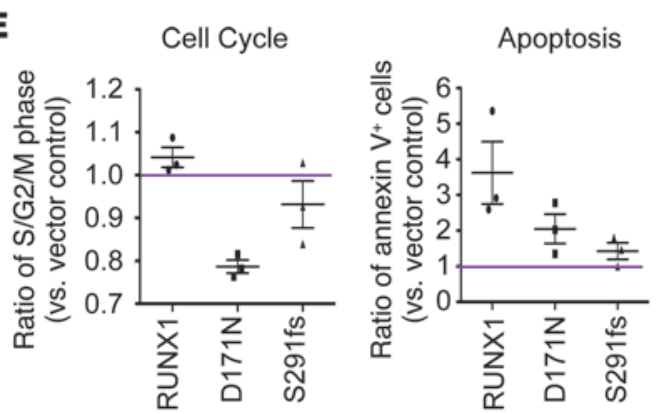

\section{Figure 1}

RUNX1 inhibits the growth of human CB cells by inducing myeloid maturation. (A) Schematic presentation of RUNX1 and its mutants. RUNT, RUNT domain; AD, transcription activation domain. (B) Expression of the individual proteins was confirmed in CB cells. (C) Changes in frequency of GFP+ cells (vector/RUNX1/mutant-expressing cells) in two independent CB cell cultures. RUNX1 and the L29S mutant inhibited the growth of CB cells, while other mutants did not. (D) CD34 and CSF2RA expression in vector/RUNX1/mutant-transduced cells on day 7 of culture. GFP+ cells were gated and analyzed. The percentage of cells in each gate $\left(C D 34^{+}\right.$or CSF2RA ${ }^{+}$cells) is indicated. (E) CB cells were transduced with vector, RUNX1, or its mutants (D171N and S291fs) and were cultured with cytokines. Cell cycle status and apoptosis were assessed on days 5 through 7 of culture. The frequency of S/G2/M phase cells in GFP+ cells (transduced cells) was first normalized to that in GFP- cells (nontransduced cells) and then was normalized to that of the vector control. For apoptosis, the frequency of annexin $\mathrm{V}+$ cells in GFP+ cells was normalized to that of the vector control. Three independent experiments were performed, and data are shown as the mean \pm SEM.

and were shown to have a dominant-negative effect over wild-type RUNX1 $(5,26,27)$. L29S was also detected in $5 \%$ of healthy people and may not be relevant to leukemogenesis (ref. 7 and Figure 1A). We confirmed the expression of each protein by immunoblotting in CB cells (Figure 1B). RUNX1 and L29S inhibited the growth of CB cells, as evidenced by the loss of GFP-expressing cells in culture. In contrast, the frequency of the cells expressing other mutants gradually increased at the late phase of culture, suggesting enhanced self-renewal of these cells (Figure 1C). We found that RUNX1expressing cells showed decreased CD34 (a stem cell marker) and increased CSF2RA (a marker for myeloid differentiation) expression, suggesting that RUNX1 induces myeloid differentiation of CD34+ cells. Leukemogenic RUNX1 mutants lost this activity (Figure 1D). In particular, S291fs-expressing cells eventually dominated the culture and grew for more than 3 months (Supplemental Discussion and Supplemental Figure 1; supplemental material available online with this article; doi:10.1172/JCI68557DS1). We also assessed cell cycle status and apoptosis on days 5 through 7 of culture in CB cells expressing RUNX1, D171N, S291fs, or vector. $\mathrm{D} 171 \mathrm{~N}$ decreased the frequency of CB cells in the S/G2/M phases, consistent with its transient inhibitory effect during the early phase of culture. Wild-type RUNX1 slightly increased the frequency of annexin $\mathrm{V}^{+}$apoptotic cells, which may also contribute to the growth-inhibitory effect of RUNX1 in CB cells (Figure 1E). Thus, RUNX1 acts as a tumor suppressor in CB cells mainly by inducing myeloid differentiation, and loss of this differentiation-inducing activity is a common feature of leukemogenic RUNX1 mutants.

Mutant RUNX1 inbibits the growth of human AML cells. Next, we transduced RUNX1 and its mutants (D171N and S291fs) into the engineered human AML cells generated by expressing AML1ETO or MLL-AF9 in human CB cells (18-20). Interestingly, both mutants inhibited the growth of these engineered AML cells (Figure 2, A and B). RUNX1 overexpression also inhibited the growth of AML1-ETO cells by inducing myeloid maturation (Figure 2, A 
A
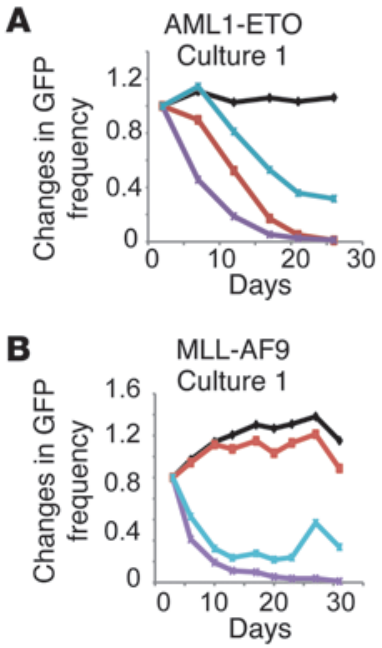
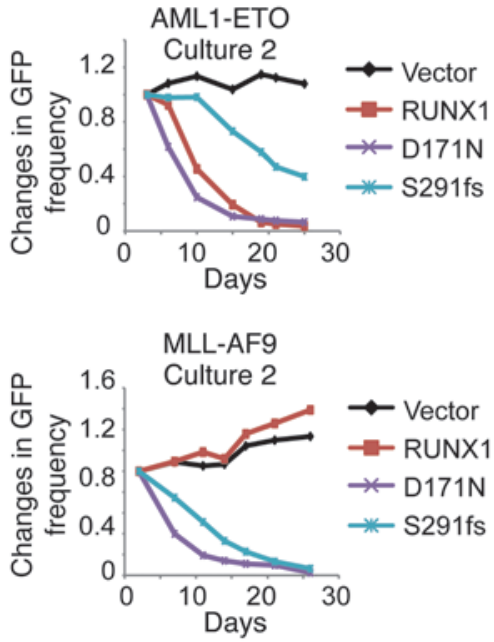

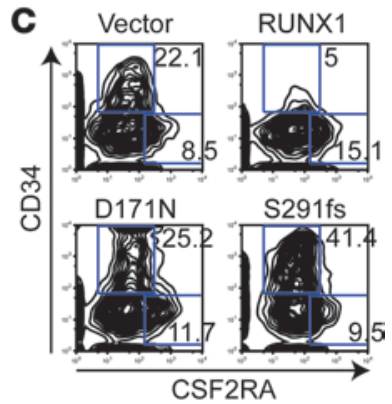

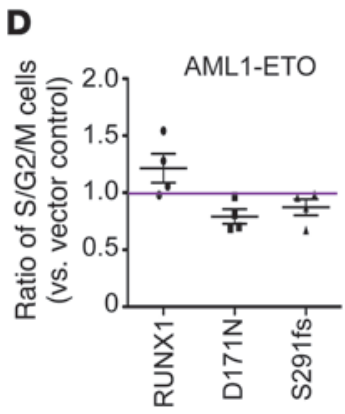
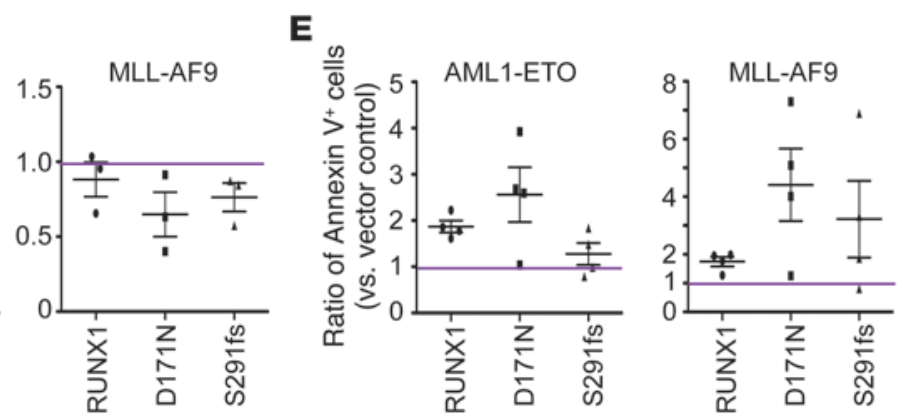

Figure 2

Mutant RUNX1 inhibits the growth of human AML cells by inducing cell cycle arrest and apoptosis. (A and B) Changes in frequency of GFP+ cells (vector/RUNX1/mutant-expressing cells) in two independent cultures of AML1-ETO (A) and MLL-AF9 cells (B). (C) CD34 and CSF2RA expression in vector/RUNX1/mutant-transduced AML1-ETO cells. The percentage of CD34+ cells in each culture is indicated. (D and E) Cells were transduced with vector, RUNX1, or its mutants (D171N and S291fs) and were cultured with cytokines. Cell cycle status and apoptosis were assessed on days 5 through 7 of culture. The frequency of S/G2/M phase cells in GFP+ cells (transduced cells) was first normalized to that in GFP- cells (nontransduced cells) and then was normalized to that of the vector control (D). For apoptosis, the frequency of annexin $\mathrm{V}^{+}$cells in GFP+ cells was normalized to that of the vector control $(\mathbf{E})$. Three or four independent experiments were performed, and data are shown as the mean \pm SEM.

and C), but the growth-inhibitory effect of RUNX1 was not seen in MLL-AF9 cells (Figure 2B). Similar to the results seen in CB cells, the mutants did not decrease CD34 expression in AML1-ETO cells (Figure 2C). Instead, the mutant-expressing AML cells showed a decrease in the proportion of S/G2/M phase cells and an increase in the proportion of annexin $\mathrm{V}^{+}$cells compared with vector-transduced cells. In particular, the mutants, but not wild-type RUNX1, induced cell cycle arrest in AML1-ETO cells and apoptosis in MLLAF9 cells (Figure 2, D and E). Because the mutant overexpression perturbs RUNX1-mediated gene regulation (Supplemental Figure 2 and Supplemental Discussion), these results indicate that proper RUNX1 function is necessary for efficient growth of these AML cells and could explain why no RUNX1 mutation was found in CBF and MLL fusion leukemias.

RUNX1 depletion inbibits the growth of human AML cells. RUNX1 was abundantly expressed and phosphorylated in all the engineered human AML cells, with the highest expression in AML1-ETO cells (Figure 3, A and B). To assess the role of endogenous RUNX1 in human cells, we knocked down RUNX1 expression in CB, in the engineered AML cells, and in myeloid cell lines using two indepen- dent RUNX1-specific shRNA lentiviruses that showed modest (sh-1) and strong (sh-2) knockdown efficiency (Figure 3C and Supplemental Figure 3A, B). We found that the reduction of RUNX1 expression resulted in substantial growth inhibition in both CB and AML cells, but the growth-inhibitory effect was relatively weak in CB cells (Figure 3D and Supplemental Figure 3C). The growth of BCR-ABLdriven leukemia cells (K562) was unaffected by RUNX1 reduction (Supplemental Figure 3D). Cell cycle and apoptosis analysis revealed a substantial decrease in the proportion of S/G2/M phase cells and an increase in the percentage of annexin $\mathrm{V}^{+}$cells in RUNX1-depleted AML1-ETO and MLL-AF9 cells (Figure 3, E and F). Consistent with the results of mutant overexpression, RUNX1 depletion blocked myeloid differentiation in CB and AML1-ETO cells, as evidenced by an increased expression of the stem cell marker CD34 (Supplemental Figure 3E). Thus, RUNX1 promotes cell cycle progression and inhibits apoptosis in AML cells, thereby supporting their growth.

Reintroduction of RUNX1 reverses the negative effect of RUNX1 shRNAs. It has been shown that some shRNAs have off-target or nonspecific toxicity (28). We therefore examined whether the reintroduction of RUNX1 could reverse the growth-inhibitory effect 
A

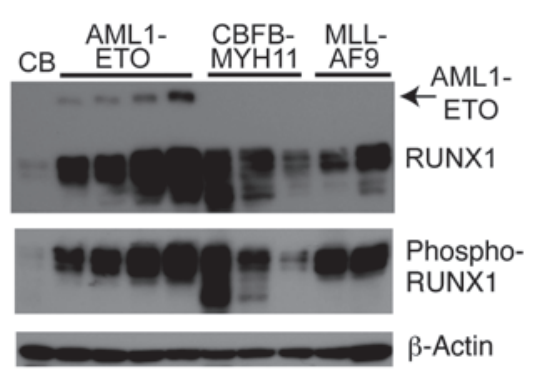

B

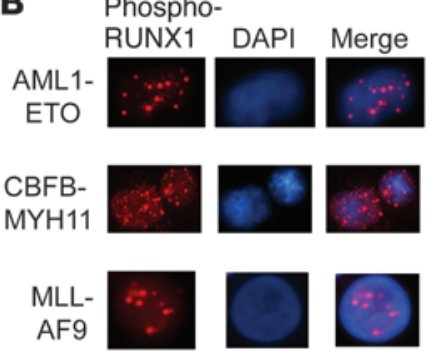

D

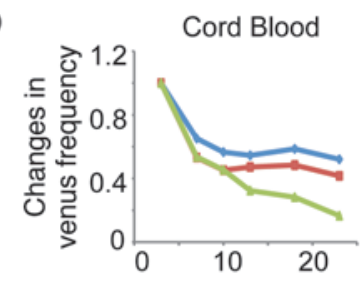

AML1-ETO
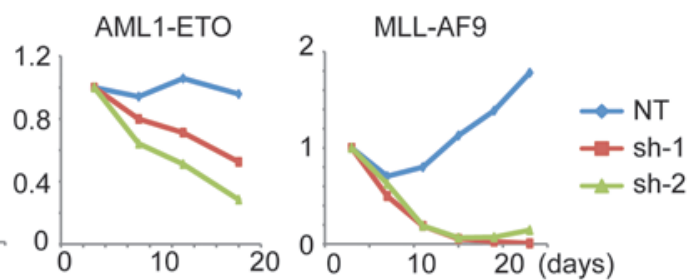
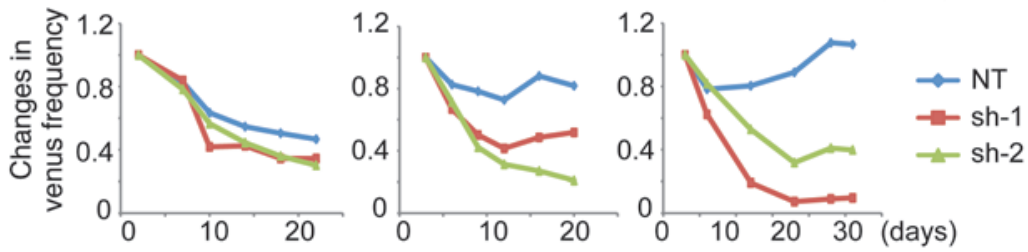

E

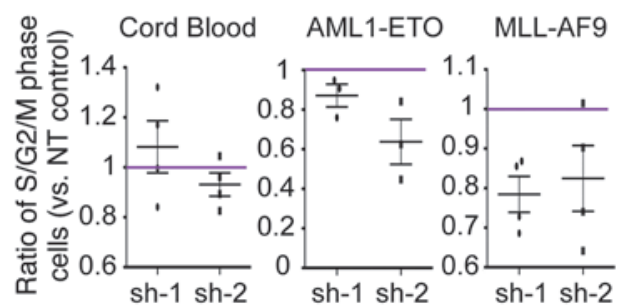

$\mathbf{F}$

Figure 3

RUNX1 depletion inhibits the growth of AML cells by inducing cell cycle arrest and apoptosis. (A) Expression and phosphorylation of RUNX1 in normal CB cells and those expressing AML1-ETO, CBFB-MYH11, or MLL-AF9. CB cells were cultured for 2 weeks, and fusion gene-expressing cells were cultured for more than 8 weeks. (B) Immunofluorescence analysis of phosphorylated RUNX1 in human AML cells. Original magnification, $\times 40$. Note that antiphospho-RUNX1 (Ser 249) detects RUNX1 but not AML1-ETO. (C) Knockdown efficiency of each shRNA was confirmed in K562 cells. See also Supplemental Figure 3B. (D) Changes in frequency of venus ${ }^{+}$cells (shRNA-transduced cells) in two independent CB, AML1-ETO, and MLL-AF9 cell cultures. (E and F) Cells were transduced with NT control or RUNX1 shRNA (sh-1 or sh-2) and were cultured with cytokines. Cell cycle status and apoptosis were assessed on days 5 through 7 of culture. For cell cycle status, the frequency of S/G2/M phase cells in venus ${ }^{+}$cells (transduced cells) was first normalized to that in venus ${ }^{-}$cells (untransduced cells) and then was normalized to that of the NT control (E). For apoptosis, the frequency of annexin $\mathrm{V}^{+}$cells in venus ${ }^{+}$cells was normalized to that of the NT control (F). Four independent experiments were performed, and data are shown as the mean \pm SEM.

of each shRNA. We cotransduced vector or RUNX1 (coexpressing GFP) with each shRNA (coexpressing venus) in MLL-AF9 cells and monitored changes in GFP/venus frequency in culture. High expression of RUNX1 in $\mathrm{GFP}^{+}$and GFP/venus double-positive (DP) populations was confirmed by immunoblotting (Figure 4A). In the vector-transduced cultures, the frequency of both venus ${ }^{+}$ and DP cells decreased on day 11 because of the growth-inhibitory effect of RUNX1 shRNAs. In contrast, RUNX1 reintroduction in the DP cells prevented this negative effect, as evidenced by the abundant DP cells in the cultures on day 11 (Figure 4B). The shRNA-resistant versions of RUNX1 also reversed the negative effect of each shRNA (Supplemental Figure 4). Furthermore, RUNX1-5A, a nonfunctional RUNX1 mutant carrying five phos- pho-deficient mutations (29), did not reverse the negative effect of sh-2, whereas all functional RUNX1 (wild-type, RUNX1-2A, and RUNX1-4A) did (Figure 4, C and D, Supplemental Figure 5, and Supplemental Discussion). Taken together, we conclude that the growth-inhibitory effect of the RUNX1 shRNAs is in fact due to RUNX1 downregulation.

Inbibiting RUNX1 function suppresses buman MLL fusion leukemia. Using MLL-AF9-expressing CB cells as a model for human AML (20), we further assessed the role for RUNX1 in the generation of AML. We cotransduced MLL-AF9 (coexpressing GFP) with a nontargeting (NT) control shRNA or RUNX1 shRNA (coexpressing venus) into $\mathrm{CD} 34^{+}$cells and cultured the cells in vitro or directly transplanted them into the NOD/SCID mice in which 
A
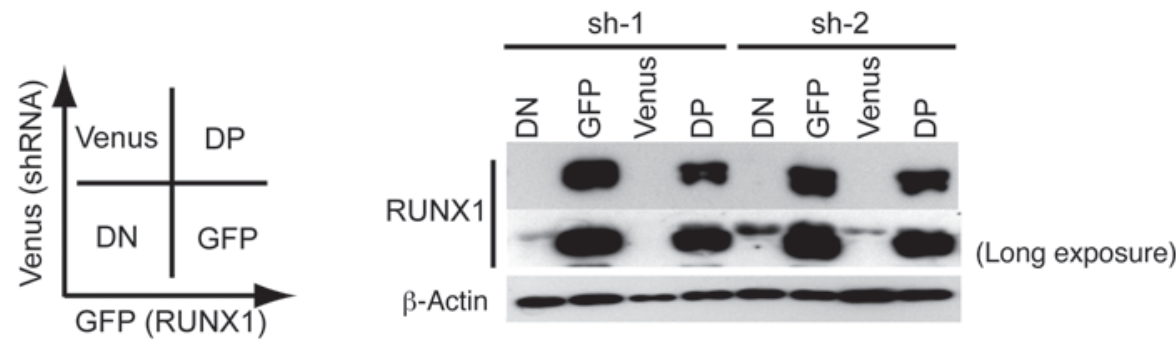

B

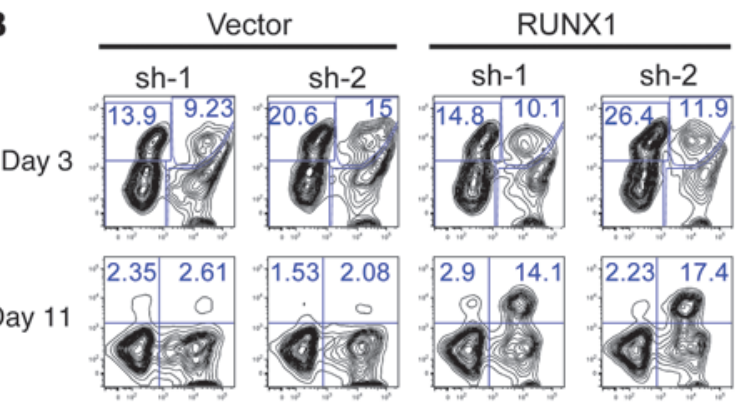

C
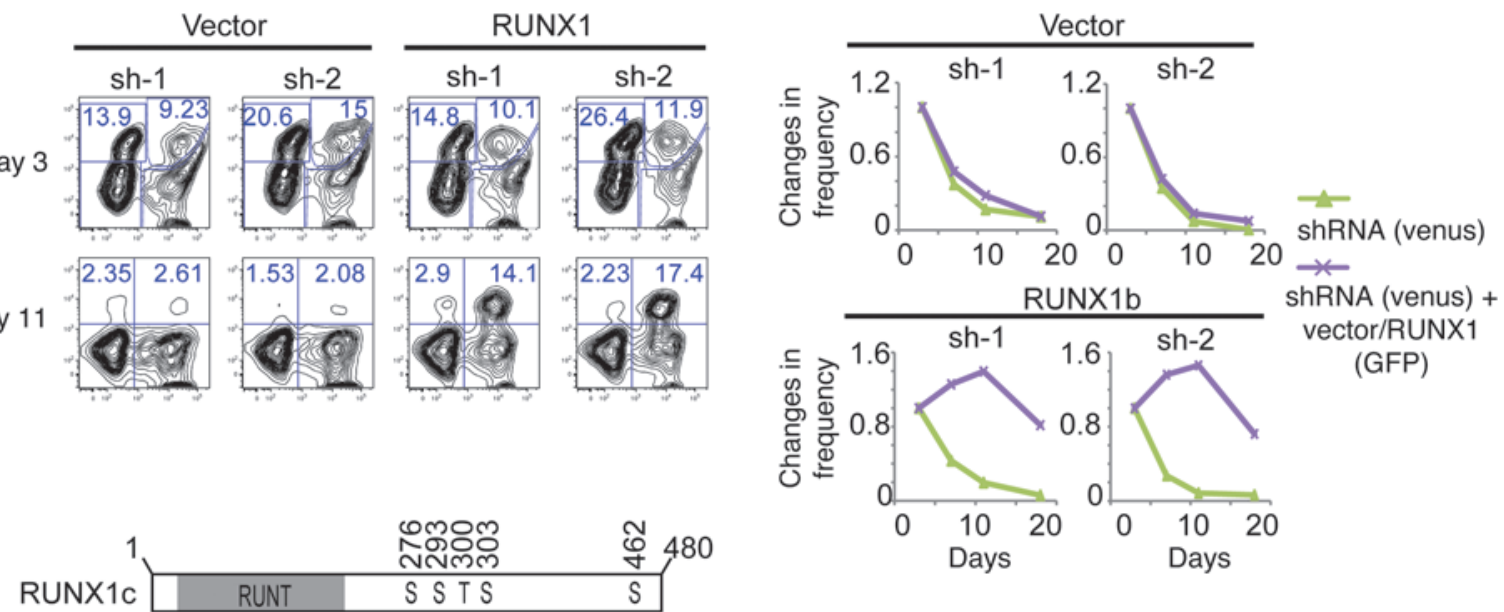

\begin{tabular}{llll|}
\cline { 3 - 4 } RUNX1C-2A & RUNT & A A T S & S \\
\cline { 2 - 3 } & RUNT & A A A A & Retain RUNX1 function \\
\cline { 2 - 3 } & RUNT &
\end{tabular}

RUNX1C-5A $\quad$ RUNT $\quad$ A A A A $\quad A \quad$ No function
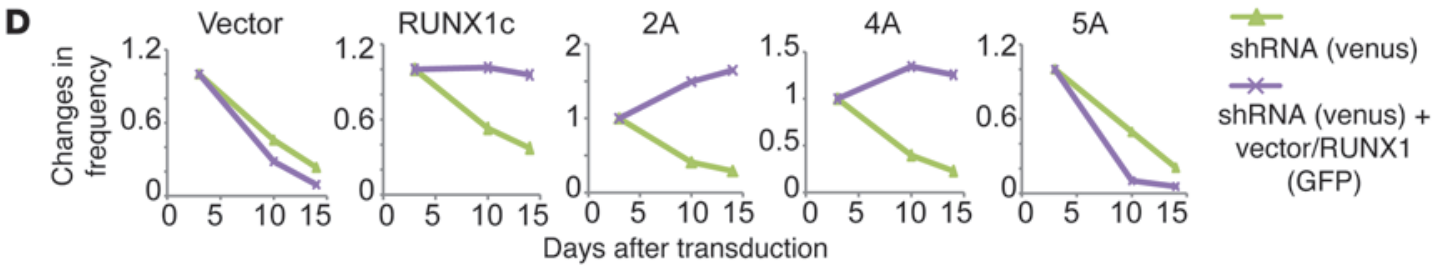

Figure 4

RUNX1 reintroduction reverses the growth-inhibitory effect of RUNX1 shRNAs. (A) MLL-AF9 cells were cotransduced with RUNX1 (GFP) and shRNAs (venus). Four distinct populations were sorted according to the GFP/venus expression (left), and RUNX1 expression in each population was assessed (right). DN/DP, GFP/venus double-negative/double-positive. (B) Expression changes of GFP (RUNX1) and venus (shRNA) in MLL-AF9 cell cultures. Left: FACS plots on day 3 (upper panels) and day 11 (lower panels) of cultures. The percentage of cells in each gate is indicated. Right: Line graphs showing changes in frequency of venus ${ }^{+}$and GFP/venus DP cells in each culture. (C) Scheme of RUNX1c (a longer isoform of RUNX1) and phosphorylation-deficient mutants with serine/threonine (S/T) to alanine (A) mutations at 2, 4, or 5 phosphorylation sites. Numbers indicate the positions of amino acid residues from the $\mathrm{N}$ terminus. (D) Changes in frequency of venus ${ }^{+}$(sh-2) and GFP/venus DP (sh-2 plus vector/RUNX1c/mutant) MLL-AF9 cells in culture. Note that RUNX1-5A is a nonfunctional mutant.

several human cytokine genes (SCF, GMCSF, and IL3) are transgenically expressed (NS-S/GM/3: NSS) (ref. 30 and Figure 5A). In these mice, MLL-AF9-expressing CB cells produced human AML within 4 to 5 weeks (Figure 5B). Consistent with earlier results, RUNX1 depletion inhibited the MLL-AF9-driven longterm proliferation of CB cells (Figure 5C). Furthermore, MLLAF9 cells transduced with RUNX1 shRNAs were outcompeted by nontransduced cells during leukemia development in vivo, as indicated by the depletion of venus ${ }^{+}$cells in leukemic mice (Figure 5D and Supplemental Figure 6A).
Next, we assessed the effect of a recently reported RUNX1 inhibitor, Ro5-3335, on the growth of MLL-AF9 cells (31). We confirmed an inhibitory effect of Ro5-3335 on human RUNX1 and RUNX2 using a CB cell culture assay (Supplemental Figure 6, B and C). We observed that the proliferation of MLL-AF9 cells was more sensitive to Ro5-3335, as compared with the proliferation of CB and K562 cells, correlating with the relative impact of RUNX1 shRNAs on the proliferation of these cells (Figure 5E). Ro5-3335 treatment induced cell cycle arrest and apoptosis in MLL-AF9 cells, similar to the effects of shRNA-mediated RUNX1 depletion (Figure 5, F and G). 
A

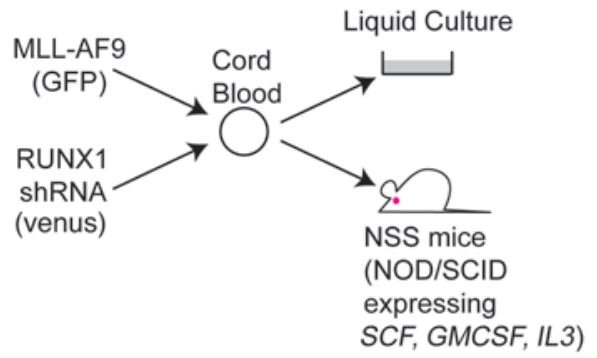

B
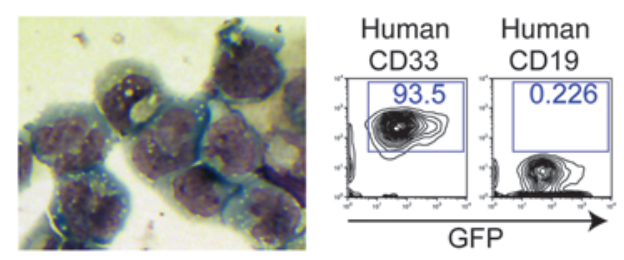

C

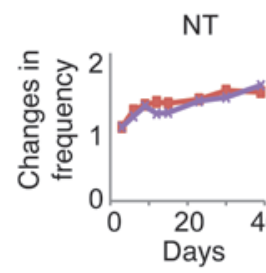

E

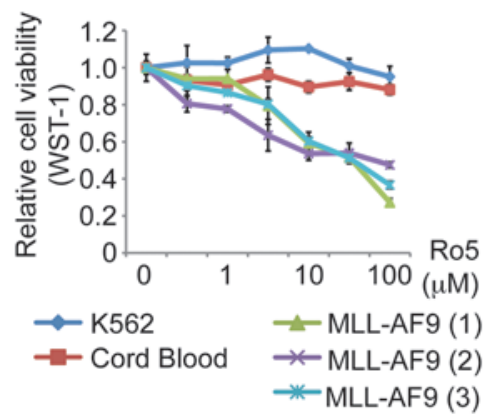

sh-1

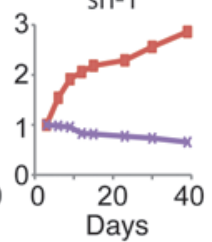

.

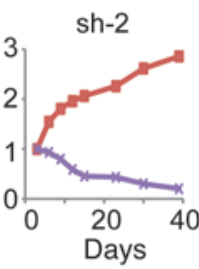

F

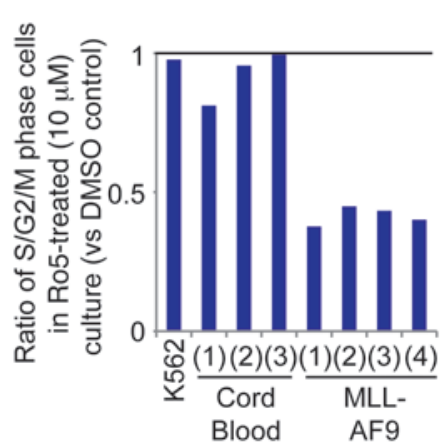

D

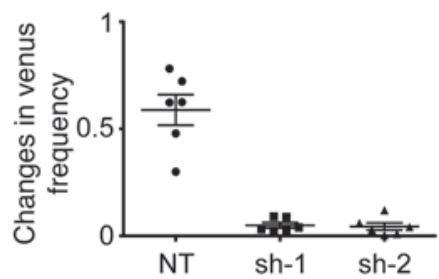

G

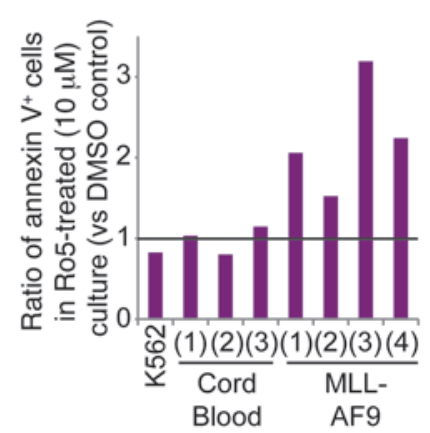

Figure 5

Suppressing RUNX1 function inhibits the development of human MLL-AF9 leukemia. (A) Experimental scheme used in B-D. (B) WrightGiemsa staining and surface expression of human antigens of human leukemia cells induced by MLL-AF9 in immunodeficient mice. The leukemia cells express human CD33 (myeloid marker) but not CD19 (lymphoid marker). Original magnification, $\times 40$. (C) Changes in frequency of GFP+ cells (MLL-AF9-expressing cells) and GFP/venus DP cells (MLLAF9/shRNA coexpressing cells) in in vitro cultures are shown as line charts. (D) Relative ratios of the venus ${ }^{+}$(shRNA-transduced) fraction in leukemic cells after transplantation compared with that before transplantation. Data are shown as the mean \pm SEM. RUNX1 shRNA-expressing (sh-1 and sh-2) venus ${ }^{+}$cells were not detected in leukemia cells ( $n=6$ for each group). See also Supplemental Figure 6A. (E) Viability of cells treated with Ro5-3335 was measured by WST-1 assay. Results are normalized to the viability of DMSO-treated cells, set at $1(n=3)$. Data are shown as the mean \pm SD of triplicate wells. (F) Relative frequency of S/G2/M phase cells in Ro5-3335-treated (10 $\mu \mathrm{M})$ cells normalized to that in DMSO-treated cells. Cells were treated with Ro53335 for 3 days. (G) Relative frequency of annexin $V^{+}$cells in Ro5-3335-treated (10 $\mu$ M) cells normalized to that in DMSO-treated cells. Cells were treated with Ro5-3335 for 3 days.

We then examined the effect of RUNX1 depletion in primary leukemia cells derived from patients with AML harboring the MLL fusions MLL-SEPT6 and MLL-ENL (Figure 6A). The AML cells with MLL-SEPT6 showed good engraftment and myeloid cell expansion in NOD/SCID/IL2 $\mathrm{rg}^{-/-}$mice expressing human $S C F$, GMCSF, and IL3 (NSG-S/GM/3: NSGS) (ref. 32 and Figure $6 \mathrm{~B}$ ). Those AML cells were transduced with NT or RUNX1 shRNAs and were then cultured on a stromal cell line to assist in the in vitro expansion of these primary leukemia cells. As expected, RUNX1 depletion inhibited the growth of the primary AML cells in vitro (Figure 6, C and D). We also transplanted the shRNA-transduced patient sample with MLL-SEPT6 directly into the NSGS mice. RUNX1-depleted AML cells showed a reduced engraftment capacity, as indicated by the reduction of the venus ${ }^{+}$fraction in human myeloid $\left(\mathrm{hCD} 45^{+} / \mathrm{hCD}^{+}\right)$cells (Figure 6D). Thus, RUNX1 supports the maintenance of human MLL fusion leukemia both in vitro and in vivo.
Functional redundancy between RUNX1 and RUNX2 in MLL fusion leukemia. In addition to RUNX1, there are two other mammalian RUNX transcription factors, RUNX2 and RUNX3, which have redundant functions in murine hematopoiesis $(33,34)$. These RUNX proteins had similar effects on human AML cells (Supplemental Figure 7). Interestingly, the rescue experiment showed that RUNX2, but not RUNX3, restored the efficient growth of RUNX1-depleted human MLL-AF9 cells, suggesting that RUNX2 has the capacity to substitute for RUNX1 in MLL fusion leukemia (Figure 7A).

Importantly, we recently showed that loss of Runx 1 accelerates the development of murine MLL-ENL leukemia using Runx1 knockout mice (11). The "growth-inhibitory" role of RUNX1 in murine MLL-ENL cells sharply contrasts with the "growth-promoting" role of RUNX1 in human MLL-AF9 cells. This discrepancy probably indicates the compensatory effect of other RUNX proteins in Runx1-deficient murine cells. Indeed, microarray data revealed an 
A

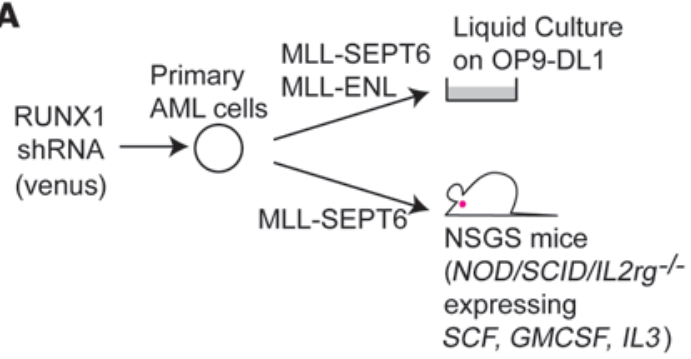

B
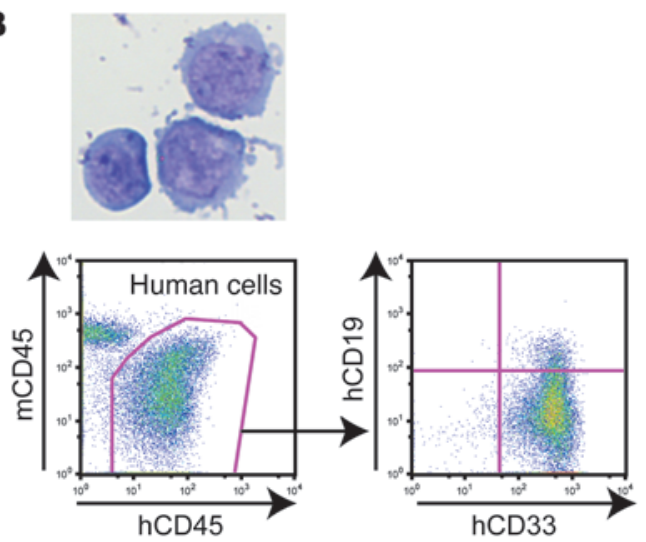

MLL-ENL
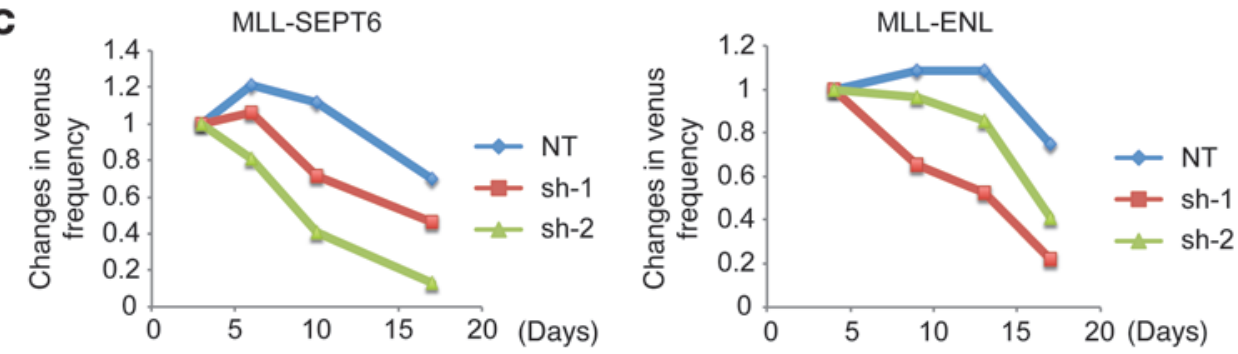

D

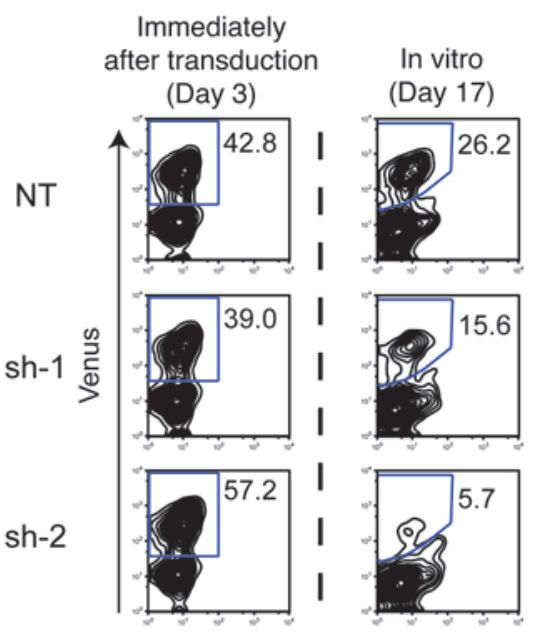

In vivo (week 6) hCD $45^{+} / \mathrm{hCD} 3^{+}$cells
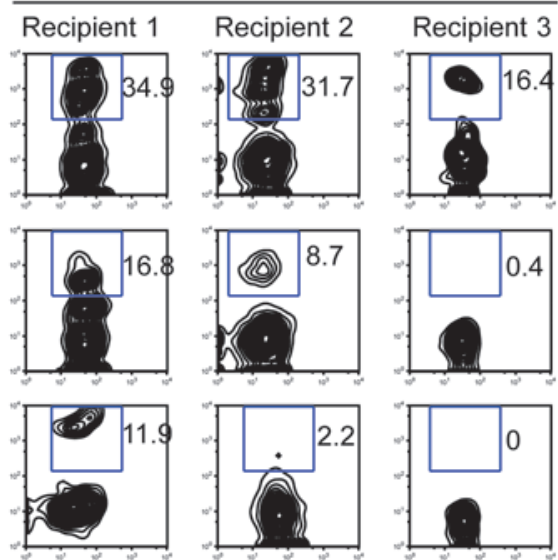

Figure 6

Suppressing RUNX1 function inhibits the growth of primary AML cells with MLL fusions. (A) Experimental scheme used in B-D. (B) WrightGiemsa staining and surface expression of primary AML cells with MLL-SEPT6 in immunodeficient mice. Original magnification, $\times 40$. $h$, human; $\mathrm{m}$, mouse. (C) Changes in frequency of venus ${ }^{+}$cells (shRNA-transduced cells) in primary AML cells with MLL-SEPT6 cells or MLL-ENL. (D) Venus expression in MLL-SEPT6 cells immediately after transduction (day 3), on day 17 in in vitro culture, and in the engrafted human myeloid cells (hCD45+/hCD33+ cells) in immunodeficient mice 6 weeks after transplantation. The percentage of venus ${ }^{+}$cells is indicated.

upregulation of Runx2 mRNA in Runx1-deficient murine MLLENL leukemia cells in vivo (Figure 7B, Supplemental Figure 8, A and B, and Supplemental Table 5). We also found an upregulation of RUNX2 protein in Runx1-deleted murine and human MLL-AF9 cells in vitro (Figure 7C and Supplemental Figure 8C). To assess the dosage-dependent function of RUNX in murine leukemia, we established an inducible gene knockout system for Runx1 and its cofactor Cbfb in MLL-AF9 cells (Figure 7D). CBFB is an important cofactor for all RUNX proteins, therefore, depletion of CBFB along with RUNX1 will result in a further reduction of RUNX activity.
The deletion of Runx1/Cbfb in MLL-AF9 cells resulted in a substantial reduction of colony numbers, while single deletion of Runx 1 showed little effect (Figure 7E). Furthermore, Runx1/Cbfb-deleted cells were gradually outcompeted by nonexcised cells, while Runx1deleted MLL-AF9 cells continued to grow even after four rounds of plating (Figure 7F).

We also performed similar experiments using a Cre-YFPexpressing retrovirus and bone marrow progenitors derived from mice with wild-type alleles, heterozygous floxed alleles of Runx1/Cbfb (Runx1/Cbfbffwt), or homozygous floxed alleles of 
A
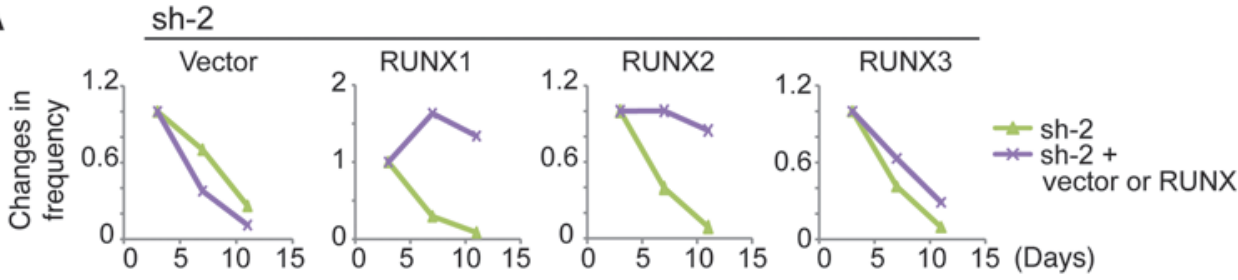

B

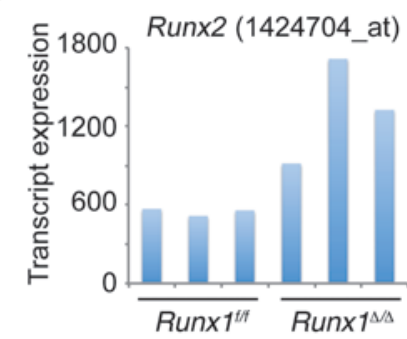

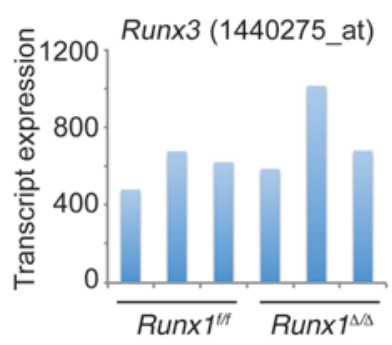

C MLL-AF9

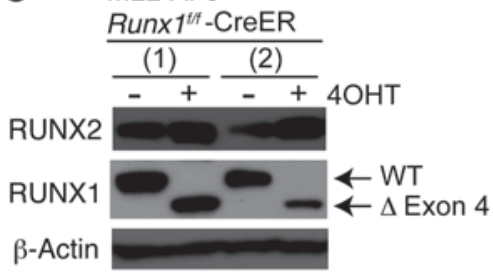

D

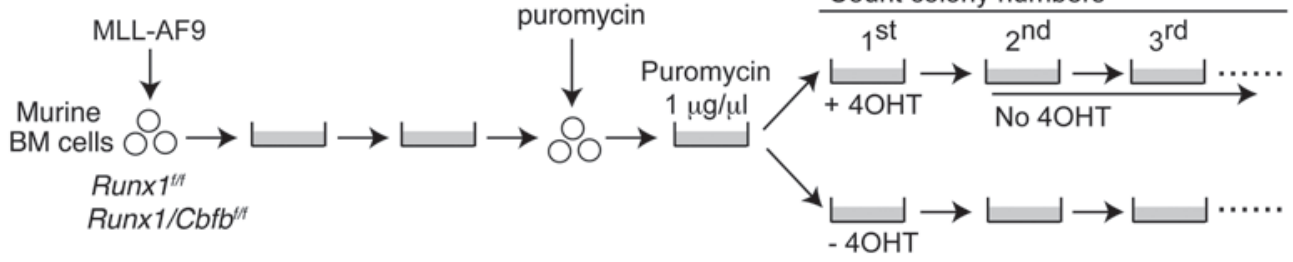

E

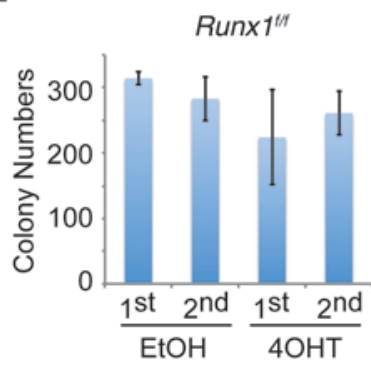

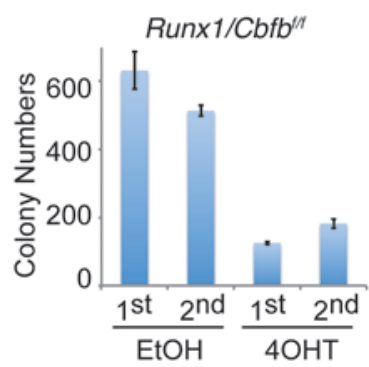

F

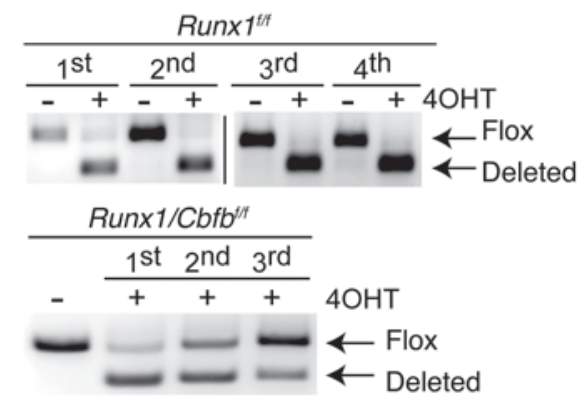

\section{Figure 7}

Functional redundancy among RUNX proteins in MLL fusion leukemia. (A) Changes in frequency of venus ${ }^{+}$(sh-2) and GFP/venus DP (shRNA plus vector or RUNX) MLL-AF9 cells in culture. (B) Expression of Runx2 and Runx3 in Runx $1^{f / f}$ and Runx $1^{\Delta / \Delta}$ murine MLL-ENL in in vivo leukemia cells. Other probes for Runx2 showed similar results (Supplemental Figure 8B). (C) Runx $1^{f / f}$ cells transduced with MLL-AF9 and CreER were treated with $\mathrm{EtOH}$ or $1 \mu \mathrm{M} 4 \mathrm{OHT}$ in $\mathrm{M} 3434$ semisolid media for 4 days and were subjected to immunoblotting with RUNX1, RUNX2, and $\beta$-actin antibodies. Cre-expressing cells produced a shorter form of RUNX1 lacking exon 4. (D) Experimental scheme used in C-F. 4OHT (1 $\mu$ M) was added only in the first round. BM, bone marrow. (E) Colony numbers are shown as the mean \pm SD from duplicate plates. (F) PCR genotyping of EtOH- or $4 \mathrm{OHT}$-treated MLL-AF9 cells for the Runx1 allele in a colony replating assay. Note that $4 \mathrm{OHT}$-treated Runx $1 / \mathrm{Cbfb}{ }^{f / f}$ cells primarily harbored a nonexcised Runx1 allele after three rounds of plating.

Cbfb or Runx1/Cbfb (Cbfffff or Runx1/Cbfff/f) (Figure 8A). Again, the combined deletion of Runx1/Cbfb in MLL-AF9 cells caused a marked reduction in colony numbers, whereas Cre expression showed marginal effects in wild-type and Runx1/Cbfbf/wt progenitors expressing MLL-AF9 (Figure 8B). Runx1/Cbfb-depleted MLLAF9 cells lost the characteristic leukemia cell morphology with prominent granules (Figure $8 \mathrm{C}$ ) and contained fewer GR-1/c-KIT double-positive cells than the controls (Figure 8D). Single deletion of $\mathrm{Cbfb}$ also moderately reduced clonogenic activity and GR-1 expression in MLL-AF9 cells (Figure 8, B and D). However, the effect of single loss of $C b f b$ was not as strong as double deletion of
Runx1/Cbfb, indicating a CBFB-independent function of RUNX1. The colony-forming activity recovered after three rounds of plating in Runx1/Cbfb-and Cbfb-depleted cultures, however; those colonies contained primarily nonexcised Runx 1 - and/or Cbfb-floxed alleles (Supplemental Figure 9).

Finally, to address whether RUNX1/CBFB are essential to maintain MLL-AF9-induced murine AML in vivo, we used a bone marrow transplant assay (Supplemental Figure 10A). Mice treated with pIpC to induce Runx1/Cbfb deletion showed less GFP ${ }^{+}$MLL-AF9 leukemia cells in peripheral blood on day 25 (Supplemental Figure 10B) and died with significantly longer latencies (Figure 8E). In contrast, pIpC 
A

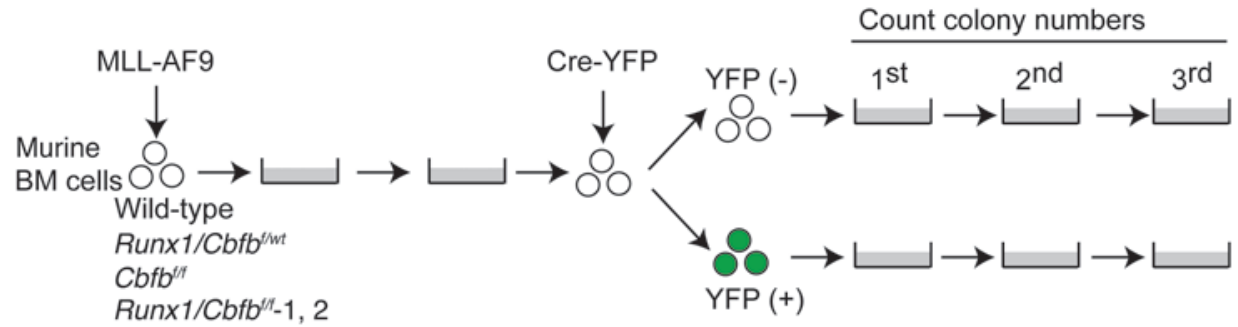

B

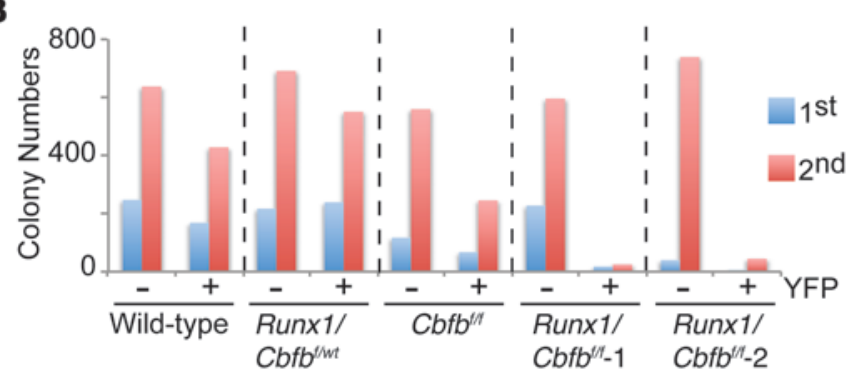

D

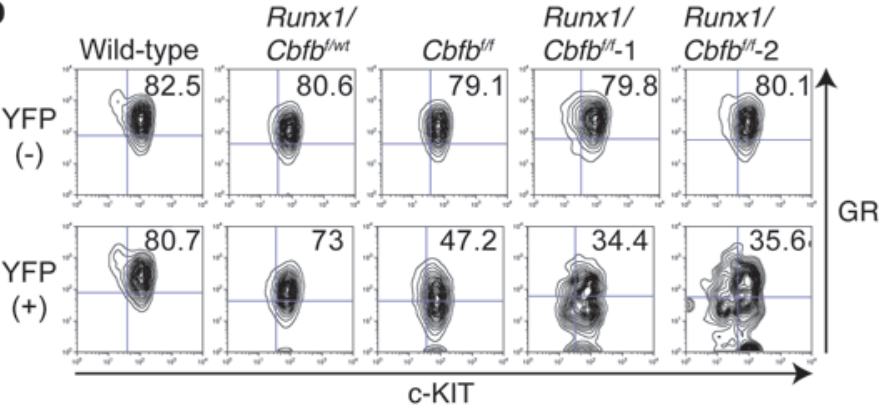

c

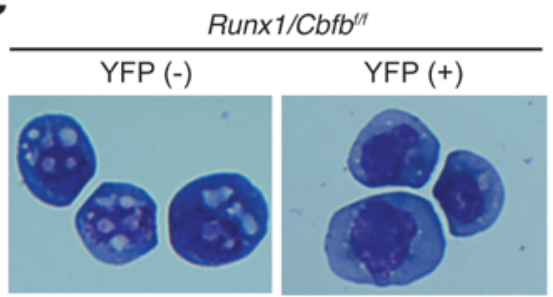

E

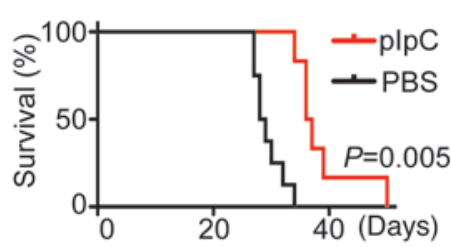

F

$\begin{aligned} & \text { Immediately } \\ & \text { after plpc }\end{aligned}$
$\begin{array}{ccc}\text { Moribund } \\ \text { mice }\end{array}$
(1) (2) (3) (1) (2)

Figure 8

Critical role of RUNX1/CBFB complex in murine MLL fusion leukemia. (A) Experimental scheme used in B-D. Murine bone marrow progenitors derived from wild-type, Runx1/Cbfb ${ }^{f / w t}, \mathrm{Cbfb}^{f / f}$, and two independent Runx1/Cbfb ${ }^{f / f}$ mice were transduced with MLL-AF9. Immortalized cells from the third to fifth rounds of in vitro plating were subsequently transduced with a Cre-YFP-expressing retrovirus. Both YFP- (nontransduced) and $\mathrm{YFP}^{+}$(Cre-transduced) cells were sorted, and then their clonogenic activity and surface marker expression were assessed in the further rounds of plating. (B) Numbers of colonies generated by Cre-transduced (YFP+) or nontransduced (YFP-) MLLAF9 cells bearing floxed alleles for Runx1, Cbfb, or both. Data are shown as the mean \pm SD from duplicate plates. (C) Wright Giemsa staining of Runx1/Cbfb-deleted (YFP+) and nondeleted (YFP-) MLL-AF9 cells. Original magnification, $\times 40$. (D) GR-1 and c-KIT expression in the indicated MLL-AF9 cells with or without Cre-YFP expression. The percentage of GR-1+/c-KIT+ cells is indicated. Loss of RUNX1/CBFB resulted in a significant reduction of GR-1+/c-KIT+ cells. Loss of CBFB also reduced GR-1 expression. (E) Survival curves of the mice transplanted with MLL-AF9 leukemia cells bearing MxCre and homozygous Runx1/Cbfb floxed alleles. Data from 8 (PBS) and 6 (plpC) mice are shown. $P=0.005$, log-rank test. (F) PCR genotyping of GFP+ MLL-AF9 cells on day 15 (immediately after plpC injection) and on day 30 from moribund mice. Excision of Runx1 was observed on day 15 , but leukemia cells on day 30 primarily harbored a nonexcised Runx1 allele.

did not affect the development of leukemia in mice transplanted with control MLL-AF9 cells (Supplemental Figure 10C). We confirmed the excised allele of Runx1 in GFP ${ }^{+}$MLL-AF9 cells immediately after $\mathrm{pIpC}$ injection, but leukemia cells harvested from moribund mice primarily harbored a nonexcised Runx1 allele (Figure $8 \mathrm{~F})$. Taken together, these results demonstrate the requirement of a certain level of RUNX activity, even in murine leukemia cells.

BCL2 contributes to the survival effect of RUNX1 in MLL fusion leukemia. To identify downstream RUNX targets, we performed global gene expression analysis in murine Runx1/Cbfbf/f MLL-AF9/CreER cells after treatment with $4 \mathrm{OHT}$ or EtOH for 24 hours (Supplemental Figure 11). This relatively short-term experiment allowed us to identify early responsive genes upon Runx1/Cbfb depletion. We confirmed the absence of RUNX1 and CBFB protein expres- sion at 24 hours. Of note, RUNX2, which was upregulated in single Runx1-deleted cells (Figure 7C), was downregulated in Runx1/ Cbfb-deleted cells (Figure 9A). This was probably due to the loss of $\mathrm{CBFB}$, which protects RUNX proteins from ubiquitin-dependent degradation (35). The dysregulated genes in Runx1/Cbfb-deleted cells contained several known RUNX1 targets, including RUNX1 itself and C/EBP $\alpha$ (Supplemental Tables 3 and 4).

Among these candidate Runx target genes, we focused on $B c l 2$, which was shown to be important for the pathogenesis of MLL fusion leukemia $(22,36)$. It was also shown that RUNX1 binds to the TGTGGT sequence in the BCL2 promoter (37). BCL2 was downregulated not only by Runx1/Cbfb double deletion, but also by single Runx1 deletion in murine MLL-AF9 cells (Figure 9B). Additional microarray data using in vivo murine MLL-ENL leu- 


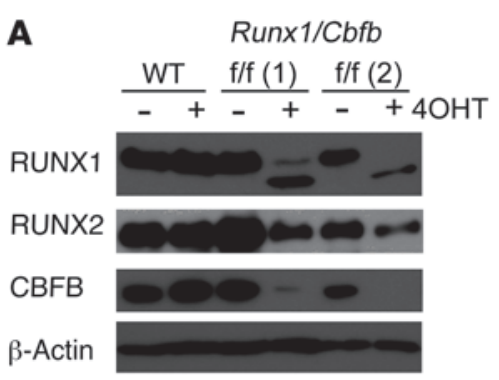

B

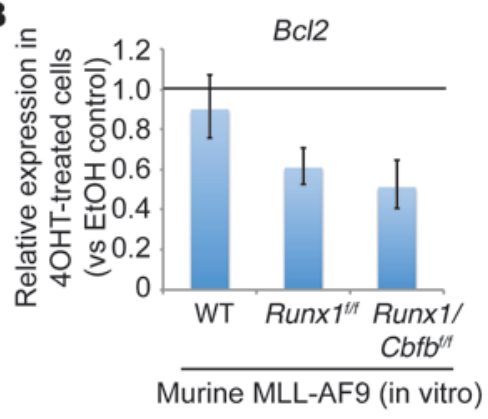

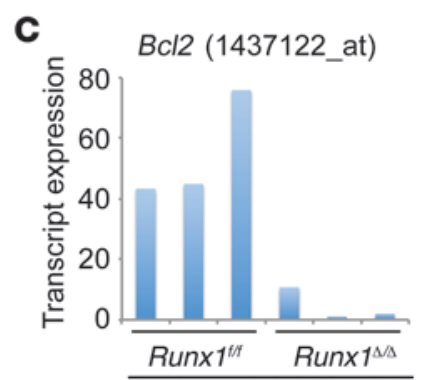

Murine MLL-AF9 (in vivo)
D

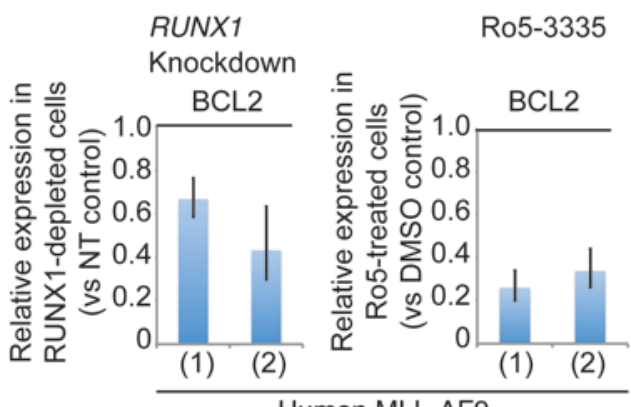

Human MLL-AF9

G

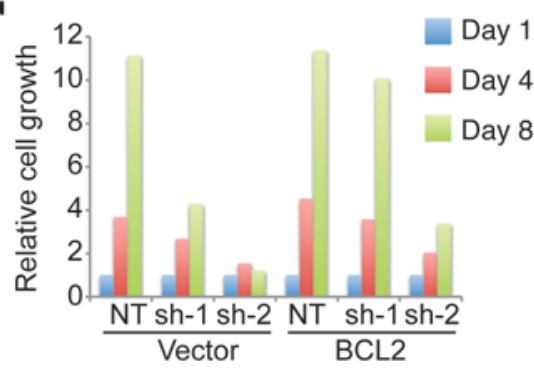

E
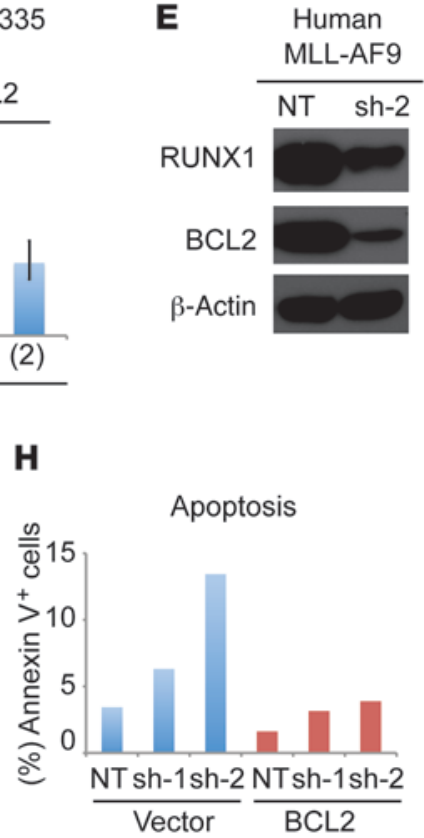

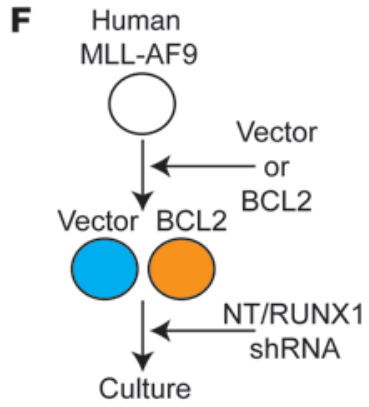

Cell Cycle

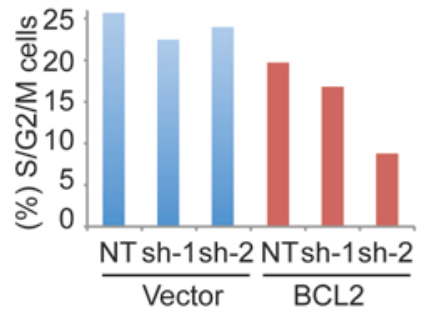

\section{Figure 9}

BCL2 antagonizes apoptosis induced by RUNX1 inhibition. (A) Immunoblotting of murine MLL-AF9/CreER-expressing cells bearing wild-type or Runx1/Cbfb floxed alleles (Runx1/Cbfb f/f) treated with EtOH or $4 \mathrm{OHT}$ for 24 hours. (B) Relative mRNA levels of Bc/2 in $4 \mathrm{OHT}$-treated wildtype, Runx $1^{1 / f}$, or Runx1/Cbfb ${ }^{f / f}$ murine MLL-AF9 cells after 4 days of treatment. Results were normalized to Gapdh, with the relative mRNA level

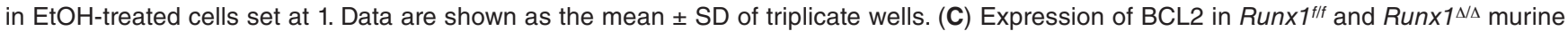
MLL-ENL in vivo leukemia cells. Other probes for BCL2 showed similar results (Supplemental Figure 12B). (D) Relative mRNA levels of BCL2 in sh-2-transduced or Ro5-3335-treated (10 $\mu \mathrm{M})$ human MLL-AF9 cells 3 days after shRNA transduction or drug addition. Results were normalized to PRKG1, with the relative mRNA level in control (NT-transduced or DMSO-treated) MLL-AF9 cells set at 1. Data are shown as the mean \pm SD of triplicate wells. (E) Protein expression of RUNX1, BCL2, and $\beta$-actin in NT or sh-2-transduced human MLL-AF9 cells. (F) Experimental scheme used in $\mathbf{G}$ and $\mathbf{H}$. See also Supplemental Figure 13. (G) Venus ${ }^{+}$(shRNA-transduced) cells $\left(1 \times 10^{6}\right)$ were sorted and cultured with cytokines. Cell numbers in each culture were counted on days 4 and 8 . (H) Apoptosis and cell cycle were assessed 4 days after shRNA transduction. Frequency of annexin $\mathrm{V}^{+}$or $\mathrm{S} / \mathrm{G} 2 / \mathrm{M}$ phase cells in each culture is shown.

kemia cells also showed substantial downregulation of BCL2 in Runx1-deficient MLL-ENL leukemia cells (Figure 9C, Supplemental Figure 12, and Supplemental Table 6). In human MLL-AF9 cells, both shRNA-mediated RUNX1 knockdown and Ro5-3333 treatment decreased BCL2 expression at mRNA and protein levels (Figure 9, D and E). To understand the role for BCL2 in RUNX1mediated cell survival, we first engineered vector or BCL2-overexpressing human MLL-AF9 cells and then assessed the effects of shRNA-mediated RUNX1 depletion in these cells (Figure 9F). BCL2-transduced MLL-AF9 cells grew better than vector-transduced cells upon RUNX1 knockdown, although BCL2-transduced cells were not fully rescued from the effects of RUNX1 depletion
(Figure 9G and Supplemental Figure 13A). BCL2-transduced MLL-AF9 cells were relatively resistant to apoptotic cell death induced by RUNX1 depletion compared with control cells. However, cell cycle arrest induced by RUNX1 depletion became more apparent in BCL2-transduced cells (Figure $9 \mathrm{H}$ and Supplemental Figure 13, B-D). As a possible explanation for this effect on the cell cycle, we found that CDKN1A was significantly upregulated in RUNX1-depleted human MLL-AF9 cells and Runx1/Cbfb-deleted mouse MLL-AF9 cells. However, CDKN1A knockdown did not rescue the growth defect of RUNX1-depleted human MLL-AF9 cells, suggesting the presence of other factors regulating RUNX1-mediated cell cycle progression (Supplemental Figure 14). 


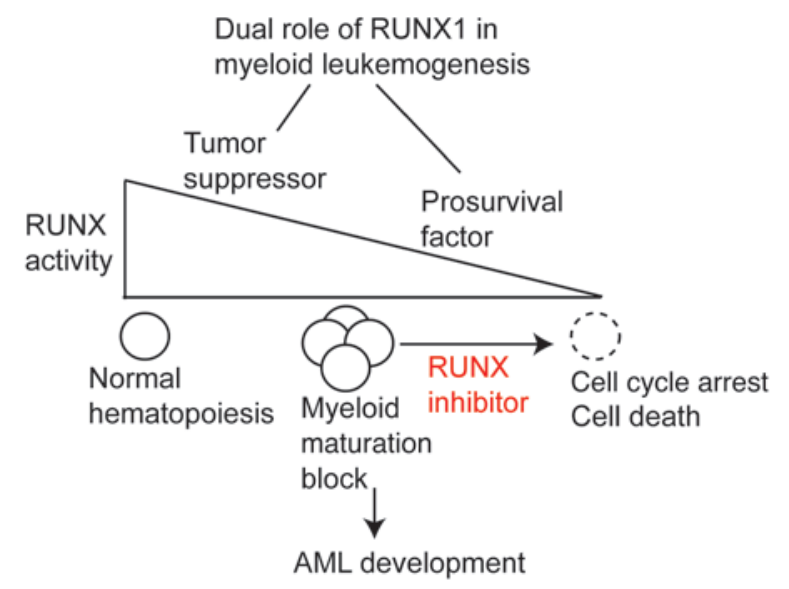

Figure 10

Proposed model illustrating dosage-dependent functions of RUNX in myeloid leukemogenesis. Partial inactivation of RUNX activity results in the expansion of myeloid progenitors and the subsequent development of AML. However, a further reduction of RUNX activity caused cell cycle arrest and cell death. AML cells will be more sensitive to RUNX inhibition because RUNX activity is already reduced in them.

\section{Discussion}

Primary human cells offer a potentially ideal target for the study of oncogene function. Using human cell-based models, we demonstrated dual functions of RUNX1 in myeloid leukemogenesis. RUNX1 overexpression inhibits the growth of CB cells by inducing myeloid differentiation, whereas a certain level of RUNX1 activity is required for the growth of AML cells. Human AML cells are more sensitive to RUNX1 inhibition probably because their RUNX activity is already reduced. We also used a mouse AML model and showed that the combined loss of RUNX1/CBFB inhibits the development of murine MLL fusion leukemia, while single loss of RUNX1 accelerates it (11). Based on these results, we propose a model to explain the dosage-dependent function of RUNX in myeloid leukemogenesis (Figure 10). Partial loss of RUNX activity blocks myeloid maturation and supports AML development, while a further reduction of RUNX activity results in cell cycle arrest and cell death.

In the past few decades, the scientific community has primarily focused on "oncogenes" that are overexpressed in tumor cells as ideal therapeutic targets. However, such oncogenes are usually also important for the maintenance of normal stem cells, and therefore it remains unclear whether inhibiting such oncogenes would favor normal cells over tumor cells. Instead, genes that are underexpressed in cancer cells but are essential for their survival will be promising targets to selectively eradicate cancer cells while preserving normal stem cells. Importantly, many so-called "tumor suppressors” appear to have such features. In addition to RUNX1, two other well-known tumor suppressors, PML and FOXOs, were shown to have survival roles in leukemia stem cells $(38,39)$. Interestingly, recent genome-wide sequence studies in myeloid neoplasms found inactivating mutations in genes that were shown to have oncogenic properties, including GATA2, EZH2, and NOTCH pathway genes (40-44). Although these findings reveal a potential tumor suppressor role for these oncogenes, it is highly likely that these genes still retain some growth-promoting function. Indeed, several recent reports showed an important role for EZH2 in the development of MLL fusion leukemia (45-47). Dosage-dependent functions of these genes in myeloid leukemogenesis need to be clarified in future studies.

Mouse models have proven to be invaluable tools for the understanding of human cancer. Nevertheless, significant differences exist between mouse models and clinical diseases. The current study highlights the potential influence on tumor development of a compensatory mechanism in mice lacking individual genes. Our data, together with a recent report showing that RUNX2 is upregulated by MLL-AF9 in mouse bone marrow cells (48), strongly indicate the functional redundancy between RUNX1 and RUNX2 in MLL fusion leukemia. The upregulated RUNX2 probably provides sufficient RUNX activity for Runx1-deficient murine MLL-ENL cells to develop leukemia. It is not clear why we did not see the compensatory effects in human cell-based models. Given that RUNX1 is not mutated in patients with MLL fusion leukemia, human MLL fusion AML cells may require stronger RUNX activity than the corresponding mouse cells for optimal growth. Alternatively, adverse effects of shRNAs used in human cells and/ or different experimental assays could affect the results. While this manuscript was under review, Wilkinson et al. showed that RUNX1 is a transcriptional target of MLL-AF4 and supports the growth of MLL-AF4 cells (49). Although they argued that RUNX1 is specifically important for the growth of MLL-AF4 cells, our study clearly demonstrates that other types of MLL fusion leukemia also require a certain level of RUNX activity. Compounds targeting RUNX/ CBFB function will show substantial efficacy in MLL fusion leukemia, and these compounds can be expected to exhibit a reasonable therapeutic window relative to normal blood stem cells.

We identified BCL2 as an important mediator for the survival effect of RUNX1 in human MLL fusion leukemia. However, BCL2 did not rescue the cell cycle arrest induced by RUNX1 depletion. Furthermore, Runx1-deficient murine MLL-AF9 cells still developed leukemia despite reduced BCL2 expression. Therefore, other factors must also contribute to the RUNX1-mediated leukemia cell growth. The mechanisms for RUNX1-mediated cell cycle progression remain elusive, but CDKN1A appears to be at least involved in this process, given its consistent upregulation in RUNX1-inhibited leukemia cells. Further characterization of RUNX/CBFB-mediated gene regulation may reveal the way to specifically block the survival function of RUNX without preventing its tumor suppressor role in promoting myeloid differentiation.

In summary, we found an unexpected survival- and growth-promoting role for RUNX1 in the development and maintenance of AML with leukemogenic fusion proteins. Recent efforts to target this transcription factor complex in CBF leukemia (31) should be extended to AML associated with MLL rearrangements and potentially to other myeloid neoplasms with RUNX1 dysfunction.

\section{Methods}

Human cell culture. CD34+ cells were separated using an EasySep CD34 selection kit (STEMCELL Technologies). CB cells and CB cells transduced with leukemia fusion genes were cultured in Iscove's modified Dulbecco's media (IMDM) containing 20\% BIT9500 (STEMCELL Technologies) and $10 \mathrm{ng} / \mathrm{ml} \mathrm{SCF}$, megakaryocyte growth and development factor (TPO), FLT3 ligand (FLT3L), IL-3, and IL-6, as described previously $(15,16)$. Primary AML cells derived from patients were cultured on a stromal cell line, OP9-DL1, with the same culture media.

Vectors and viral transduction. The retroviral constructs pMYs-IRES-EGFP (pMYs-IG), pMYs-RUNX1b-IG, pMYs-RUNX1b/D171N-IG, and pMYs- 
RUNX1b/S291fs-IG were provided by T. Kitamura (Institute of Medical Science, University of Tokyo, Tokyo, Japan). RUNX1b/R139G, RUNX2, and RUNX3 cDNAs were provided by M. Kurokawa (University of Tokyo, Tokyo, Japan), and we cloned them into pMYs-IG. These cDNAs were fused with a FLAG epitope tag at the N terminus. RUNX1b-L378fs and RUNX1b-L29S were obtained using mRNA prepared from a myeloid leukemia cell line, CG-SH (27), and were inserted into the pMYs-IG. RUNX1c and its phospho-deficient mutants (2A, 4A, and 5A) were provided by A.S. Kraft (The Medical University of South Carolina, Charleston, South Carolina, USA) and cloned into pMYs-IG. For MLL-AF9 expression, we used pMSCV-MLL-AF9-pgk-EGFP or pMSCV-MLL-AF9-pgk-puro. The pBabe-IRES-puro, pBabe-BCL2-IRESpuro, and pMSCV-CreERT2-puro constructs were from Addgene (http://www. addgene.org). Lentiviral vector MISSION pLKO.1-shRNA-puro constructs targeting human RUNX1 (TRCN0000013659 [sh-1] and TRCN0000013660 [sh-2]) (Supplemental Figure 3) were obtained from Sigma-Aldrich. The venus marker was excised from the PLKO.1-venus construct with BAMH1 and KPN1 and was subcloned into the corresponding sites on the RUNX1 shRNAs to replace the puromycin-resistant gene. Viral production was performed by transfecting viral plasmids along with gag-, pol-, and env-expressing plasmids into $293 \mathrm{~T}$ cells, as described previously $(17,24)$.

Flow cytometry. Cells were analyzed on a FACSCanto I, II, or III flow cytometer (BD). Antibodies for flow cytometric analysis were all purchased from BD Biosciences (Supplemental Table 1). Cells were stained with fluorochrome-conjugated antibodies incubated for 30 minutes at $4{ }^{\circ} \mathrm{C}$ and were washed with $2 \%$ FBS in PBS prior to analysis. Cell cycle analysis (Vybrant DyeCycle Violet stain; Invitrogen) and apoptosis analysis (Annexin V-APC kit; BD Biosciences) were performed according to the manufacturer's recommendations.

Western blotting and immunofluorescence. Western blot analysis was performed as described previously (21). Detection was performed using SuperSignal West Pico or Femto Chemiluminescent Substrate (Pierce, Thermo Scientific). For immunofluorescence, cytospins were fixed with paraformaldehyde and permeabilized with Triton X-100 and then incubated with primary antibodies, followed by labeling with Alexa Fluor 568-conjugated anti-rabbit antibody (Molecular Probes). Nuclei were visualized with DAPI (SlowFade Gold Antifade; Invitrogen). Cells were visualized on a Zeiss Axiovert $200 \mathrm{M}$ fluorescent microscope with a Zeiss Plan-Apochromat $63 \times / 1.4$ objective (Carl Zeiss). Data were recorded with an ORCA-ER digital camera (Hamamatsu). Deconvolution was performed with OpenLab 4.0.3 software (Improvisions). The antibodies used are shown in Supplemental Table 1.

Quantitative PCR. RNA was isolated with the RNeasy kit (QIAGEN). RNA was reversed transcribed using MuLV RT and random hexamers (Applied Biosystems). cDNA was then subjected to quantitative PCR using SYBR Green or TaqMan technology (Applied Biosystems). The primers used are shown in Supplemental Table 2.

Drug studies. Human MLL-AF9 cells, CD34+ CB, and K562 cells were plated with titrating doses of Ro5-3335 (ranging from $0 \mu \mathrm{M}$ to $100 \mu \mathrm{M}$ ) in triplicate. After 72 hours, $10 \mu \mathrm{lWST}-1$ cell proliferation assay premix (MK 400; Takara Bio Inc.) was added to each well. Plates were read at 450 to $560 \mathrm{~nm}$ to measure optical density. Cells treated with $10 \mu \mathrm{M}$ Ro5-3335 for 72 hours were analyzed for cell cycle status and apoptosis using flow cytometry, as described above.

In vivo xenotransplantation assay. Human $\mathrm{CB} \mathrm{CD} 34^{+}$cells were transduced with MLL-AF9 (coexpressing GFP) and shRNAs (coexpressing venus), and were injected intrafemorally into sublethally irradiated (250 cGy from a cesium source) 6- to 8-week-old NSS mice immediately after transduction. Approximately $3 \times 10^{5} \mathrm{CB}$ cells were injected into a recipient mouse. Primary AML cells harboring MLL-SEPT6 were transduced with venus-expressing shRNAs and were injected into NSGS mice using the same method. Approximately $1 \times 10^{6} \mathrm{AML}$ cells were injected into a recipient mouse. The immunodeficient mice were placed on doxycycline chow (TestDiet; Modified Prolab RMH-1500 with 0.0625\% doxycycline) prior to, during, and for several weeks after irradiation.

Gene-targeted mice. The mice harboring a Runx 1 allele with exon 4 flanked by loxP sites $\left(R u n x 1^{f / f}\right)(50)$ and the mice harboring a Cbfb allele with exon 5 flanked by loxP sites (Cbfbff) (51) were crossed to generate Runx1/Cbfb/ff mice. Another Runx1 mutant mouse strain with loxP-flanked exon 5 (52) was used to generate in vivo MLL-ENL leukemia (11).

Myeloid colony assay. Murine myeloid colony assays were plated in M3434 cytokine-enriched methylcellulose according to the manufacturer's instructions (STEMCELL Technologies). CreER-expressing cells were selected in M3434 containing $1 \mu \mathrm{g} / \mathrm{ml}$ puromycin. Cells $\left(5 \times 10^{3}\right)$ were plated for each round of plating. Colony counting and replating were performed every 4 or 5 days.

Mouse bone marrow transplantation assay. Bone marrow progenitors (c-KIT ${ }^{+}$ cells) were transduced with MLL-AF9 and were injected into sublethally irradiated (700 Gy) recipient mice via the tail vein. Secondary transplantation was performed in the same way using $1 \times 10^{6}$ spleen cells isolated from leukemic mice.

Gene expression analysis. Total RNA was prepared using an RNeasy Mini Kit (QIAGEN). For microarray analysis with MLL-AF9/CreER-expressing Runx1/ $\mathrm{Cbfb/f}$ cells, the quality of the total RNA was checked by an Agilent Bioanalyzer 2100 (Hewlett Packard) using the RNA 6000 Nano Assay. For each sample, the Ambion WT Expression Kit (Life Technologies) synthesized the cDNA target from 300 nanograms of total RNA. Then the GeneChip WT Terminal Labeling Kit (Affymetrix) was used to both chemically fragment and biotin label the cDNA target. Labeled probes were hybridized to the GeneChip Mouse Gene 1.0 ST Array (Affymetrix) and were scanned with the Affymetrix GeneChip Scanner 3000 7G. Raw data files were created by Command Console, the Affymetrix operating software program. All samples were normalized using the robust multichip average (RMA). Upregulated or downregulated genes in Runx1/Cbfb-deleted cells were identified using Qlucore Omics Explorer (http://www.qlucore.com). For microarray analysis with Runx 1 fff-and Run $\times 1^{\Delta / \Delta-M L L-E N L}$ in vivo leukemia cells, labeling of fragmented cDNA was done using the NuGen Ovation Biotin labeling system (NuGEN Technologies). Labeled probes were hybridized to the Mouse Genome 4302.0 Array (Affymetrix) and scanned with a GeneChip Scanner $30007 \mathrm{G}$ (Affymetrix). The expression data were extracted from the image files produced with GeneChip 1.0 Operating software (Affymetrix). Normalization and expression value calculations were performed using the DNA Chip Analyzer (www.dchip.org) (53). Microarray data were deposited in the NCBI's Gene Expression Omnibus (GEO accession numbers GSE47350 and GSE47402).

Statistics. The survival distributions (Figure 8E) were compared using a logrank test. A $P$ value of less than 0.05 was considered statistically significant.

Study approval. Human umbilical CB cells and patient samples were obtained from the Translational Trials Development and Support Laboratory at Cincinnati Children's Hospital Medical Center (Cincinnati, Ohio, USA) in accordance with IRB-approved protocols. Written informed consent was obtained in accordance with the Declaration of Helsinki. All mouse experiments were performed under an IACUC-approved protocol of the Cincinnati Children's Hospital in accordance with accepted national standards and guidelines.

\section{Acknowledgments}

We thank the Flow Cytometry Core, the Mouse Core, and the Microarray Core at Cincinnati Children's Hospital Medical Center for their help. This work was supported by an Institutional Clinical and Translational Science Award; an NIH/NCRR grant (1UL1RR026314-01); a Translational Trials Development and Support Laboratory award (USPHS, MO1 RR 08084); a Center of Excel- 
lence in Molecular Hematology P30 award (DK090971); a grant from the CancerFree Kids Foundation for Cancer Research (to J.C. Mulloy and S. Goyama); a grant from the Intramural Research Program of the National Human Genome Research Institute, NIH (to P.P. Liu); and a JSPS Postdoctoral Fellowship for Research Abroad (to S. Goyama). J.C. Mulloy is a Leukemia and Lymphoma Society Scholar. The contents of the manuscript are solely the responsibility of the authors and do not necessarily represent the official views of the NIH.
Received for publication December 26, 2012, and accepted in revised form June 20, 2013.

Address correspondence to: James C. Mulloy, Division of Experimental Hematology and Cancer Biology, Cincinnati Children's Hospital Medical Center, University of Cincinnati College of Medicine, Cincinnati, Ohio 45229, USA. Phone: 513.636.1844; Fax: 513.636.3768; E-mail: james.mulloy@cchmc.org.
1. Link KA, Chou FS, Mulloy JC. Core binding factor at the crossroads: determining the fate of the HSC. J Cell Physiol. 2010;222(1):50-56.

2. Kurokawa M. AML1/Runx1 as a versatile regulator of hematopoiesis: regulation of its function and a role in adult hematopoiesis. Int J Hematol. 2006;84(2):136-142.

3. Goyama S, Mulloy JC. Molecular pathogenesis of core binding factor leukemia: current knowledge and future prospects. Int J Hematol. 2011;94(2):126-133.

4. Huang G, et al. The ability of MLL to bind RUNX1 and methylate $\mathrm{H} 3 \mathrm{~K} 4$ at PU.1 regulatory regions is impaired by MDS/AML-associated RUNX1/AML1 mutations. Blood. 2011;118(25):6544-6552.

5. Harada H, Harada Y, Niimi H, Kyo T, Kimura A, Inaba $\mathrm{T}$. High incidence of somatic mutations in the AML1/RUNX1 gene in myelodysplastic syndrome and low blast percentage myeloid leukemia with myelodysplasia. Blood. 2004; 103(6):2316-2324.

6. Kohlmann A, et al. Next-generation sequencing technology reveals a characteristic pattern of molecular mutations in $72.8 \%$ of chronic myelomonocytic leukemia by detecting frequent alterations in TET2, CBL, RAS, and RUNX1. J Clin Oncol. 2010;28(24):3858-3865.

7. Tang JL, et al. AML1/RUNX1 mutations in 470 adult patients with de novo acute myeloid leukemia: prognostic implication and interaction with other gene alterations. Blood. 2009;114(26):5352-5361.

8. Schnittger S, et al. RUNX1 mutations are frequent in de novo AML with non complex karyotype and confer an unfavourable prognosis. Blood. 2011;117(8):2348-2357.

9. Motoda L, et al. Runx1 protects hematopoietic stem/progenitor cells from oncogenic insult. Stem Cells. 2007;25(12):2976-2986.

10. Jacob B, et al. Stem cell exhaustion due to Runx1 deficiency is prevented by Evi5 activation in leukemogenesis. Blood. 2010;115(8):1610-1620.

11. Nishimoto N, et al. Loss of AML1/Runx1 accelerates the development of MLL-ENL leukemia through down-regulation of p19ARF. Blood. 2011;118(9):2541-2550.

12. Patel JP, et al. Prognostic relevance of integrated genetic profiling in acute myeloid leukemia. $N$ Engl JMed. 2012;366(12):1079-1089.

13. Kamikubo Y, et al. Accelerated leukemogenesis by truncated CBF beta-SMMHC defective in high-affinity binding with RUNX1. Cancer Cell. 2010;17(5):455-468.

14. Hyde RK, et al. Cbfb/Runx1 repression-independent blockage of differentiation and accumulation of Csf2rb-expressing cells by Cbfb-MYH11. Blood. 2010;115(7):1433-1443.

15. Wunderlich M, Mulloy JC. Model systems for examining effects of leukemia-associated oncogenes in primary human CD34+ cells via retroviral transduction. Methods Mol Biol. 2009;538:263-285.

16. Mulloy JC, Wunderlich M, Zheng Y, Wei J. Transforming human blood stem and progenitor cells: a new way forward in leukemia modeling. Cell Cycle. 2008;7(21):3314-3319.

17. Wunderlich M, Krejci O, Wei J, Mulloy JC. Human CD34+ cells expressing the inv(16) fusion protein exhibit a myelomonocytic phenotype with greatly enhanced proliferative ability. Blood.
2006;108(5):1690-1697.

18. Mulloy JC, et al. Maintaining the self-renewal and differentiation potential of human $\mathrm{CD}_{3} 4^{+}$hematopoietic cells using a single genetic element. Blood. 2003;102(13):4369-4376.

19. Mulloy JC, Cammenga J, MacKenzie KL, Berguido FJ, Moore MA, Nimer SD. The AML1-ETO fusion protein promotes the expansion of human hematopoietic stem cells. Blood. 2002;99(1):15-23.

20. Wei J, et al. Microenvironment determines lineage fate in a human model of MLL-AF9 leukemia. Cancer Cell. 2008;13(6):483-495.

21. Krejci O, et al. p53 signaling in response to increased DNA damage sensitizes AML1-ETO cells to stress-induced death. Blood. 2008;111(4):2190-2199.

22. Mizukawa B, et al. Inhibition of Rac GTPase signaling and downstream prosurvival Bcl-2 proteins as combination targeted therapy in MLL-AF9 leukemia. Blood. 2011;118(19):5235-5245.

23. Zuber J, et al. RNAi screen identifies Brd4 as a therapeutic target in acute myeloid leukaemia. Nature. 2011;478(7370):524-528.

24. Chou FS, et al. The THPO/MPL/Bcl-xL pathway is essential for survival and self-renewal in human pre-leukemia induced by AML1-ETO. Blood. 2012;120(4):709-719.

25. Ng KP, et al. p53 independent epigenetic-differentiation treatment in xenotransplant models of acute myeloid leukemia. Lenkemia. 2011;25(11):1739-1750.

26. Imai $Y$, et al. Mutations of the AML1 gene in myelodysplastic syndrome and their functional implications in leukemogenesis. Blood. 2000;96(9):3154-3160.

27. Munker R, et al. Characterization of a new myeloid leukemia cell line with normal cytogenetics (CGSH). Leuk Res. 2009;33(10):1405-1408.

28. Grimm D, et al. Fatality in mice due to oversaturation of cellular microRNA/short hairpin RNA pathways. Nature. 2006;441(7092):537-541.

29. Yoshimi M, et al. Multiple phosphorylation sites are important for RUNX1 activity in early hematopoiesis and T-cell differentiation. Eur J Immunol. 2012;42(4):1044-1050.

30. Feuring-Buske M, Gerhard B, Cashman J, Humphries RK, Eaves CJ, Hogge DE. Improved engraftment of human acute myeloid leukemia progenitor cells in beta 2-microglobulin-deficient NOD/SCID mice and in NOD/SCID mice transgenic for human growth factors. Leukemia. 2003;17(4):760-763.

31. Cunningham $L$, et al. Identification of benzodiazepine Ro5-3335 as an inhibitor of CBF leukemia through quantitative high throughput screen against RUNX1-CBFbeta interaction. Proc Natl Acad Sci U S A. 2012;109(36):14592-14597.

32. Wunderlich $M$, et al. AML xenograft efficiency is significantly improved in NOD/SCID-IL2RG mice constitutively expressing human SCF, GM-CSF and IL-3. Lenkemia. 2010;24(10):1785-1788.

33. Goyama $\mathrm{S}$, et al. The transcriptionally active form of AML1 is required for hematopoietic rescue of the AML1-deficient embryonic para-aortic splanchnopleural (P-Sp) region. Blood. 2004;104(12):3558-3564.

34. Fukushima-Nakase Y, Naoe Y, Taniuchi I, Hosoi $\mathrm{H}$, Sugimoto T, Okuda T. Shared and distinct roles mediated through $\mathrm{C}$-terminal subdomains of acute myeloid leukemia/Runt-related transcription factor molecules in murine development. Blood.
2005;105(11):4298-4307.

35. Huang G, Shigesada K, Ito K, Wee HJ, Yokomizo T, Ito Y. Dimerization with PEBP2beta protects RUNX1/AML1 from ubiquitin-proteasome-mediated degradation. EMBO J. 2001;20(4):723-733.

36. Dawson MA, et al. Inhibition of BET recruitment to chromatin as an effective treatment for MLL-fusion leukaemia. Nature. 2011;478(7370):529-533.

37. Klampfer L, Zhang J, Zelenetz AO, Uchida H, Nimer SD. The AML1/ETO fusion protein activates transcription of BCL-2. Proc Natl Acad Sci U S A. 1996;93(24):14059-14064.

38. Sykes SM, et al. AKT/FOXO signaling enforces reversible differentiation blockade in myeloid leukemias. Cell. 2011;146(5):697-708.

39. Ito $\mathrm{K}$, et al. PML targeting eradicates quiescent leukaemia-initiating cells. Nature. 2008; 453(7198):1072-1078.

40. Klinakis A, et al. A novel tumour-suppressor function for the Notch pathway in myeloid leukaemia. Nature. 2011;473(7346):230-233

41. Nikoloski G, et al. Somatic mutations of the histone methyltransferase gene EZH2 in myelodysplastic syndromes. Nat Genet. 2010;42(8):665-667.

42. Ernst $T$, et al. Inactivating mutations of the histone methyltransferase gene EZH2 in myeloid disorders. Nat Genet. 2010;42(8):722-726.

43. Hahn CN, et al. Heritable GATA2 mutations associated with familial myelodysplastic syndrome and acute myeloid leukemia. Nat Genet. 2011;43(10):1012-1017.

44. Ostergaard P, et al. Mutations in GATA2 cause primary lymphedema associated with a predisposition to acute myeloid leukemia (Emberger syndrome). Nat Genet. 2011;43(10):929-931.

45. Neff T, et al. Polycomb repressive complex 2 is required for MLL-AF9 leukemia. Proc Natl Acad Sci US A. 2012;109(13):5028-5033.

46. Tanaka S, et al. Ezh2 augments leukemogenicity by reinforcing differentiation blockage in acute myeloid leukemia. Blood. 2012;120(5):1107-1117.

47. Shi J, et al. The Polycomb complex PRC2 supports aberrant self-renewal in a mouse model of MLL-AF9;Nras(G12D) acute myeloid leukemia. Oncogene. 2012;32(7):930-938.

48. Bernt KM, et al. MLL-rearranged leukemia is dependent on aberrant H3K79 methylation by DOT1L. Cancer Cell. 2011;20(1):66-78.

49. Wilkinson AC, et al. RUNX1 is a key target in $t(4 ; 11)$ leukemias that contributes to gene activation through an AF4-MLL complex interaction. Cell Rep. 2013;3(1):116-127.

50. Growney JD, et al. Loss of Runx1 perturbs adult hematopoiesis and is associated with a myeloproliferative phenotype. Blood. 2005;106(2):494-504.

51. Naoe Y, et al. Repression of interleukin- 4 in $T$ helper type 1 cells by Runx/Cbf beta binding to the Il4 silencer. J Exp Med. 2007;204(8):1749-1755.

52. Ichikawa M, et al. AML-1 is required for megakaryocytic maturation and lymphocytic differentiation, but not for maintenance of hematopoietic stem cells in adult hematopoiesis. Nat Med. 2004;10(3):299-304.

53. Li C, Hung Wong W. Model-based analysis of oligonucleotide arrays: model validation, design issues and standard error application. Genome Biol. 2001;2(8):RESEARCH0032. 